DR. MASSIMILIANO RUSCICA (Orcid ID : 0000-0002-0195-7061)

Article type : Review

NAFLD OR MAFLD DIAGNOSES AND CARDIOVASCULAR DISEASES: FROM EPIDEMIOLOGY TO

\title{
DRUG APPROACHES
}

P. Dongiovanni ${ }^{1}$, E. Paolini ${ }^{1,2}$, A. Corsini ${ }^{2,3}$, C.R. Sirtori ${ }^{2}$ and M. Ruscica ${ }^{2}$

${ }^{1}$ General Medicine and Metabolic Diseases; Fondazione IRCCS Cà Granda Ospedale Maggiore Policlinico, Milan, Italy; ${ }^{2}$ Department of Pharmacological and Biomolecular Sciences, Università degli Studi di Milano, Milan, Italy; ${ }^{3}$ Multimedica IRCCS, Sesto San Giovanni (MI), Milan, Italy.

\section{Corresponding Authors}

Massimiliano Ruscica

Department of Pharmacological and Biomolecular Sciences

Università degli Studi di Milano

e-mail: massimiliano.ruscica@unimi.it

Paola Dongiovanni

Fondazione IRCCS Cà Granda Ospedale Maggiore Policlinico

e-mail: paola.dongiovanni@policlinico.mi.it

This article has been accepted for publication and undergone full peer review but has not been through the copyediting, typesetting, pagination and proofreading process, which may lead to differences between this version and the Version of Record. Please cite this article as doi: 10.1111/ECI.13519

This article is protected by copyright. All rights reserved 
Background: A consensus of experts has proposed to replace the term nonalcoholic fatty liver disease (NAFLD), whose global prevalence is $25 \%$, with metabolic dysfunction-associated fatty liver disease (MAFLD), to describe more appropriately the liver disease related to metabolic dysfunction. MAFLD is closely intertwined with type 2 diabetes, obesity, dyslipidemia, all linked to a rise in the risk of cardiovascular disease (CVDs). Since controversy still stands on whether or not NAFLD/MAFLD raises the odds of CVD, the present review aims to evaluate the impact of NAFLD/MAFLD etiologies on CV health and the potential correction by dietary and drug approaches.

Results: Epidemiological studies indicate that NAFLD raises risk of fatal or non-fatal CVD events. NAFLD patients have a higher prevalence of arterial plaques and stiffness, coronary calcification, and endothelial dysfunction. Although genetic and environmental factors strongly contribute to NAFLD pathogenesis, a Mendelian randomization analysis indicated that the PNPLA3 genetic variant leading to NAFLD may not be causally associated with CVD risk. Among other genetic variants related to NAFLD, TM6SF2 appears to be protective, whereas MBOAT7 may favor venous thromboembolism.

Conclusions: NAFLD is correlated to a higher CVD risk which may be ameliorated by dietary interventions. This is not surprising, since new criteria defining MAFLD include other metabolic risk abnormalities fueling development of serious adverse extrahepatic outcomes, e.g., CVD. The present lack of a targeted pharmacological approach makes the identification of patients with liver disease at higher CVD risk (e.g., diabetes, hypertension, obesity or high levels of C-reactive protein) of major clinical interest.

Keywords: cardiovascular risk, MAFLD, NAFLD, PNPLA3, PCSK7, PCSK9, TM6F2, MBOAT7

This article is protected by copyright. All rights reserved 


\section{Introduction}

Over the past two decades, nonalcoholic fatty liver disease (NAFLD) has raised the burden of death and disability caused by cardiovascular diseases (CVDs) ${ }^{1}$. The prevalence of NAFLD in adults is $25 \%$ (95\% Cl: $22.10-28.65)$ worldwide, the highest being reported in the Middle East (32\%) and South America (31\%), followed by Asia (27\%), USA (24\%) and Europe (23\%), with the lowest in Africa (14\%) ${ }^{2,3}$. Considering that NAFLD and CVD share common risk factors such as atherogenic dyslipidemia, insulin resistance (IR), and hypertension, the overall aim of the present review is to cover aspects common to the two conditions, along with the description of new pharmacological approaches to NAFLD that could ameliorate CVD risk. However, due to the very recent recommendation to switch the terminology NAFLD into metabolic dysfunction-associated fatty liver disease (MAFLD), this aspect has been taken into consideration in the research strategy and throughout the manuscript. By using the Pubmed database, the following algorithm has been used: NAFLD OR MAFLD AND adipose tissue AND alcohol consumption AND cardiovascular disease AND dietary approach AND ectopic fat AND epigenetic AND genetic determinants AND inflammation AND insulin resistance AND pharmacological treatment AND subclinical atherosclerosis AND type 2 diabetes mellitus. Relative to clinical studies the search for literature comprised observational, retrospective, interventional and prospective studies. PD and MR screened titles and full text of papers identified in our search.

Although not in the remit of the present review article it is worth mentioning that NAFLD is also associated with an increased risk of other cardiac complications (valvular calcification, left ventricular hypertrophy and certain arrhythmias) independent of common CVD risk factors ${ }^{4}$.

NAFLD associates also with a raised risk of liver-related morbidity or mortality, and is considered a multisystem disorder, affecting a variety of extra-hepatic organs, including the CV system ${ }^{5}$. The largest meta-analysis ( $n=34,043$ adult individuals) of observational, prospective and retrospective studies indicated that patients with NAFLD have an odds ratio (OR) of 1.64 (95\% Cl 1.26-2.13) for fatal and/or non-fatal CVD events. This risk increases stepwise with the progression of the disease, leading to an OR of $2.58(95 \% \mathrm{Cl} 1.78-3.7 \%)$ in patients with the most severe NAFLD phenotype ${ }^{6}$. In a similar way, a closer link between NAFLD and subclinical atherosclerosis has been reported: the presence of NAFLD raises the odds of carotid-intima 
media thickness (c-IMT), arterial stiffness and coronary artery calcification by $1.74(95 \% \mathrm{Cl} 1.47-$ 2.06), 1.56 (95\% Cl1.24-1.96), and 1.40 (95\% Cl 1.22-1.60), respectively ${ }^{7}$.

\section{Metabolic fatty liver disease (MAFLD) - a novel acronym}

Several years ago, concerns over the accuracy of the fatty liver disease nomenclature were raised 8, encouraging renaming this definition. Indeed, a consequence of using inappropriate terminology may generate mistrust between patients and clinicians and most importantly terminology that contains the term "non" may diminish the importance of a disease leading to a false perception ${ }^{9}$. NAFLD terminology can be considered as just an umbrella term, essentially targeting a single feature, i.e., hepatic steatosis. It is a condition of "exclusion" since it exists only in the absence of conditions such as viral hepatitis, autoimmune disease or alcohol intake. Conversely, manifestation of fatty liver should be the final sum of different interactions, from circulating lipids, to IR to genetic background ${ }^{10}$. The term NAFLD could be an element of confusion since the use of "non" and of words such as "alcoholic" is disliked by patients leading to a fear of stigma, especially in pediatric conditions ${ }^{11}$. All these criticisms have been the cue to find a terminology addressing the heterogenous clinical presentation of the disease. In 2020, a consensus of experts has proposed to replace the term NAFLD with a more appropriate one, i.e., MAFLD ${ }^{12}$. This new nomenclature should allow to properly stratify patients via the application of more precise genetic, anthropometric, and metabolic approaches. This new definition clearly establishes this disease as a metabolic disorder. As depicted in Figure 1, to meet the diagnosis of MAFLD, patients require the presence of hepatic steatosis (histology, imaging, blood markers or scores of evidence of fat accumulation) accompanied by one of the following three features: overweight or obesity (cut-offs according to the ethnicity), type 2 diabetes mellitus (T2D) or signs of metabolic dysregulation. This last is defined as the presence of two or more conditions: (1) enlarged waist circumference; (2) raised blood pressure or specific drug treatment; (3) raised triglycerides (TG) or specific drug treatment; (4) low high-density lipoprotein cholesterol (HDL-C); (5) prediabetes; (6) high Homeostatic Model Assessment of IR (HOMA-IR) score; (7) inflammation with raised levels of high-sensitivity C-reactive protein (hSCRP) ${ }^{12}$. Overall, the ability of these non-invasive scoring models to discriminate between NAFLD and MAFLD has to be validated in future studies ${ }^{13}$, as in the case of serum biomarkers of fatty liver that could replace imaging 
methods. Currently, should the epidemiological studies be conducted or analyzed again, the former assumption appears to be valid for markers with a high prognostic score, e.g., fatty liver index ${ }^{14}$.

This new definition is challenging and probably could lead to different conclusions when applied to previous studies. On this matter, a recent study on 756 Japanese patients with fatty liver demonstrated that the application of the MAFLD criteria identifies individuals with fatty liver and significant fibrosis, with a higher sensitivity for detecting significant fibrosis in MAFLD than NAFLD, i.e., $93.9 \%$ and $73.0 \%$, respectively ${ }^{15}$. Similar conclusions have been reached when the two definitions have been used to characterize 13,083 cases with complete ultrasonographic and laboratory data. Compared with those with NAFLD, patients diagnosed as MAFLD were more likely to have multiple metabolic comorbidities with more frequent advanced fibrosis ${ }^{16}$. Moreover, as highlighted by Targher, while these real-world data show an excellent concordance between MAFLD and NAFLD (Cohen's kappa coefficient of 0.92), the two terms are not mutually interchangeable and do not identify exactly the same individuals ${ }^{17}$.

\section{Risk factors contributing to NAFLD/MAFLD}

Considering the multifactorial etiology of fatty liver, the next sections will address the contribution of genetic and epigenetic factors, ethnicity, age, sex, body composition, dietary habits, as well as changes in microbiota composition on the development of NAFLD/MAFLD. As reported by the International expert consensus statement, MAFLD could take into consideration, in the future, also pathophysiological modifiers leading to the development of a morphologically limited set of histological features. This could be the presence of the genetic variants for Patatinlike Phospholipase Domain-Containing 3 (PNPLA3), Transmembrane 6 Superfamily Member 2 gene (TM6SF2), Membrane Bound O-acyltransferase Domain-containing 7 (MBOAT7) and Hydroxysteroid 17-Beta Dehydrogenase 13 (HSD17B13), as well as epigenetic and or other disease modifiers ${ }^{14}$.

3.1 Genetic contribution. NAFLD is a complex disease whose pathogenesis and progression result from gene-environment interactions ${ }^{18}$. Familial, twin and epidemiological studies indicate that NAFLD has a strong inherited component, ranging from 20 to $75 \%$ depending on ethnicity, 
environmental factors and methodology. Family members of overweight children with NAFLD have a higher susceptibility to develop fatty liver compared to family members of obese children without NAFLD ${ }^{19}$. In 313 Finnish twins, $60 \%$ of the variation in serum alanine aminotransferase $(A L T)$, a marker of liver fat content, was genetically determined ${ }^{20}$. The concept of inheritance was further confirmed by Loomba et al who reported that the presence of hepatic steatosis and fibrosis was correlated between monozygotic but not between dizygotic twins ${ }^{21}$. Conversely, a negligible role for the heritability of NAFLD was found in a cohort of 208 adult Hungarian twins (63 monozygotic and 41 dizygotic pairs); in this series NAFLD was associated with carotid plaque formation and raised C-IMT 22.

Single nucleotide polymorphisms (SNPs) in genes regulating the hepatic lipid handling have been broadly associated with increased susceptibility to develop the entire spectrum of NAFLD, from steatosis, to nonalcoholic steatohepatitis (NASH) and fibrosis (Figure 2). Hepatic fat accumulation represents the main driver of the progression to end-stage liver damages in genetically predisposed individuals and the effect of each genetic variation on the spectrum of NAFLD is closely intertwined with the ability to induce fat storage ${ }^{23}$. The dominant genetic modifiers known to shape both NAFLD susceptibility and progression are the variants in PNPLA3, TM6SF2, MBOAT7, Glucokinase regulator (GCKR) and 17 $\beta$-HSD13 genes (Figure 3).

3.1.1 PNPLA3. The rs738409 C>G polymorphism in the PNPLA3 gene, encoding the aminoacid substitution of isoleucine to methionine at position 148 (p.I148M), accounts for the largest fraction of genetic predisposition to NAFLD and for the entire spectrum of related progressive liver damage ${ }^{24,25}$. It was identified in 2008 by a genome-wide association study (GWAS) evaluating a North American population of different ethnicities (Dallas Heart Study). The prevalence of the $G$ at-risk allele was higher in Hispanics (49\%) than in Europeans (23\%) and less frequent in African Americans (17\%), thereby explaining the inter-ethnic susceptibility to NAFLD 26. PNPLA3 is mainly localized in the endothelial reticulum (ER) and at the lipid droplet surface in hepatocytes, adipocytes and hepatic stellate cells (HSCs) where it exerts a hydrolyzing activity towards TG and retinyl esters ${ }^{27}$. While the wild-type protein is rapidly degraded, the mutated form escapes ubiquitination and accumulates on lipid droplets, thus impairing TG mobilization by other lipases (ATGL/PNPLA2), as well as turnover and catabolism 28. Another potential 
mechanism explaining TG accumulation in the presence of the p.I148M variant is impaired lipophagy in hepatocytes. This mechanism may hamper autophagic fluxes and lipid droplet degradation ${ }^{29}$.

A lipidomic analysis demonstrated that TG in very-low density lipoproteins (VLDL) were depleted of polyunsaturated fatty acids (PUFAs) in I148M homozygotes both under fasting and postprandial conditions ${ }^{30}$. By using in vitro models of I148M hepatic cells, the same authors demonstrated a raised PUFA incorporation into TG, whilst PUFA-containing diacylglycerols (DAGs) accumulated at the expense of phosphatidylcholines ${ }^{30}$. The hepatic lipid composition of DAG species may affect insulin sensitivity, although the alteration in liver DAG levels was not confirmed by Franko et al. who concluded that the PNPLA3 variant is tightly associated with fatty liver, but not with IR, thereby dissociating these two features ${ }^{31}$.

Another intriguing aspect regards ceramide synthesis, a process identified as the key mediator of hepatic IR. Since ceramide enriched liver lipidome was observed in IR-related NAFLD but not in PNPLA3 NAFLD, this may explain why metabolic NAFLD and not PNPLA3-related NAFLD is tightly correlated with an increased risk of T2D and CV events ${ }^{32}$.

Furthermore, the I148M variant impairs retinol release from HSCs, directly participating in fibrogenesis and carcinogenesis, independent of predisposition to fatty liver ${ }^{24}$. NAFLD patients who carry the $G$ allele are characterized by the activation of hepatic stem/progenitor cell $(\mathrm{HpSC})$, associated with a more aggressive histological pattern (portal fibrogenesis) and oxidative stress 33.

3.1.2 TM6SF2. In 2014, an exome wide association study identified the rs58542926 C>T genetic variant in the transmembrane 6 superfamily member 2 gene (TM6SF2) as a determinant of hepatic TG content, serum aminotransferases, low-density lipoprotein cholesterol (LDL-C) and TG. It encodes the substitution of the loss-of-function lysine (E) with glutamic acid (K) at position 167 (E167K) ${ }^{34}$. TM6SF2 localizes in the ER and ER-Golgi compartments and participates in hepatic VLDL lipidation and assembly in the ER cisternae. The E167K variant causes a TM6SF2 misfolded protein undergoing rapid intracellular turnover and degradation thus leading to hepatic down-regulation ${ }^{34}$. 
The E167K genetic variant is independently associated with higher circulating levels of ALT, hepatic TG content, and NAFLD stages in both children and adults ${ }^{35,36}$. Another exome-wide association study showed that, among 444 coding and noncoding genetic variants associated with plasma lipids, the E167K TM6SF2 variant was related to an increased risk of fatty liver and T2D ${ }^{37}$. In line with these findings, in a large cross-sectional cohort of 1,201 individuals with biopsy-proven NAFLD, the E167K variant was associated with a higher degree of steatosis, necroinflammation, ballooning and fibrosis, but was protective against CV events ${ }^{36}$. Therefore, it has been suggested that the T risk allele may disentangle NAFLD from CV disorders, while increasing liver disease severity ${ }^{36,38}$.

Liver lipidome analyses in NAFLD carriers of the E167K variant showed that hepatic TG and cholesteryl ester (CE) were higher, whereas phosphatidylcholines (PCS) were lower. In addition, incorporation of PUFA into TG and PC in TM6SF2 knockdown hepatocytes was reduced, suggesting that impairment in liver lipid synthesis from PUFAs could contribute to a deficiency in PCs and increased intrahepatic TG in E167K TM6SF2 carriers ${ }^{39}$.

3.1.3 MBOAT7. In 2015, a GWAS found that the common rs641738 C>T variant in the MBOAT7TMC4 locus on chromosome 19 increased susceptibility to cirrhosis in alcoholics ${ }^{40}$. Mancina and Dongiovanni further demonstrated that the rs641738 variant associates with liver fat accumulation and to the whole phenotypic spectrum of liver injuries related to NAFLD ${ }^{41}$, as also confirmed in pediatric NAFLD ${ }^{42,43}$.

MBOAT7, known as lyso-phosphatidylinositol (lyso-PI) acyl-transferase1 (LPIAT1), encodes an enzyme member of the Lands' cycle of phospholipid acyl-chain remodeling in membranes. It is mainly localized in the membrane bridging ER and mitochondria where fat biosynthesis and lipid droplet formation occur. MBOAT7 conjugates an acyl-CoA to the second acyl-chain of lysophospholipids, using arachidonoyl-CoA as the substrate. Thus, it modulates desaturation of phospholipids and availability of free arachidonic acid, the precursor of proinflammatory eicosanoids. These mechanisms underlying the association between the rs641738 variant and liver damage are related to the hampered hepatic gene and protein expression of MBOAT7, thus altering the phosphatidylinositol species ${ }^{41,44} 45$. An impairment in MBOAT7 function contributes to the accumulation of saturated phospholipids, mainly phosphatidylinositol species that may be 
shunted to the synthesis of saturated and mono-unsaturated TG, further contributing to fattyladen hepatocyte formation ${ }^{44,46}$.

3.1.4 GCKR. Alongside PNPLA3, TM6SF2 and MBOAT7 variants, even the common loss-offunction rs1260326 C>T variant in the GCKR gene (c.1403C >T, p.P446L) has been associated with increased fasting TG concentrations, large VLDL, steatosis and liver damage ${ }^{47}$. The GCKR gene codifies for the GCKR protein, that exerts a crucial role in glucose homeostasis regulating glucose influx into hepatocytes and activating de novo lipogenesis (DNL).

Santoro et al. reported for the first time the rate of DNL by determining incorporation of deuterium into palmitate in VLDL after administration of a carbohydrate drink ( $75 \mathrm{~g}$ glucose and $25 \mathrm{~g}$ fructose) in obese adolescents. In these individuals, the GCKR rs1260326 variant in homozygosity increased liver lipid synthesis as the result of an enhanced glycolytic carbon flux to TG formation. Moreover, the combination of PNPLA3 and GCKR minor alleles may explain up to $32 \%$ of the liver fat content in Caucasian obese children, $39 \%$ in African-Americans and $15 \%$ in Hispanics ${ }^{35}$. Furthermore, this variant has been also associated with increased susceptibility to NASH, fibrosis and hepatocellular carcinoma (HCC) 48 in adult NAFLD patients, without altering the atherogenic lipid profile and the CV risk ${ }^{49}$.

3.1.5 HSD17B13. In 2018, an exome-wide sequencing identified the rs72613567 variant in the hydroxysteroid 17-beta dehydrogenase 13 (HSD17B13) gene, associated with protection against histological steatohepatitis, clinically significant fibrosis and cirrhosis, in both NAFLD and alcoholic fatty liver disease (ALD) ${ }^{50}$. The rs72613567 variation corresponds to an insertion of an adenine adjacent to the donor splice site of the last exon (TA allele), resulting in a truncated transcript, reduced expression and impaired enzyme activity of the HSD17B13 protein ${ }^{51}$. Hsd17b13 KO mice have an impaired hepatic-lipid metabolism, resulting in raised hepatic TG content, directly inducing DNL through sterol regulatory element-binding protein (SREBP)1 and fatty acid synthase ${ }^{51,52}$.

The HSD17B13 protective effect is more relevant in the development of steatohepatitis than in progression to fibrosis ${ }^{47}$. The likely protective mechanism of the HSD17B13 rs 143404524 polymorphism appears to be an increased concentration of hepatic phospholipids that couples 
with a down-regulation of pro-inflammatory genes ${ }^{53}$. Furthermore, the rs72613567 variant has been related to a reduced risk of elevated transaminases and HCC 54,55.

3.2 Allelic pleiotropy: how genetic variants related to NAFLD affect CV risk. A genetic variant may influence multiple traits through a mechanism defined as "allelic pleiotropy" 56 . A metaanalysis of 7,176 NAFLD individuals of European descent showed that the rs780094 T allele at GCKR was associated with higher levels of LDL-C, TG and increased 2 hours glycemia. The E167K TM6SF2 variation was instead related to lower LDL-C and TG levels, whereas the I148M PNPLA3 variant was not associated with any of these traits ${ }^{57}$. The conclusion that carriers of the rs738409 PNPLA3 variant have a raised risk of NALFD progression but not of IR or metabolic syndrome highlights the conclusion that not "all forms of NAFLD were created equal" 58. Conversely, an association between I148M PNPLA3 (95\%Cl 1.03-1.07) and E167K TM6SF2 (95\%Cl 1.05-1-12) polymorphisms and diabetes was found in a fine mapping study, which evaluated 81,412 T2D cases and 370,832 controls of diverse ancestry, further confirming the contribution of the GCKR P446L variant (OR 1.05; 95\%Cl 1.04-1.07) on IR ${ }^{59}$.

Relative to coronary artery disease (CAD), a GWAS meta-analysis of $\sim 185,000$ cases and controls, showed an association of the PNPLA3 G-allele rs738409 with a modest protection from CAD, albeit significant only by running a recessive model (the odd was $0.92(95 \% \mathrm{Cl} 0.87-0.97))^{60}$ (Table 1). Thus, it is tempting to speculate that a possible cardioprotective effect may be related to the reduced VLDL secretion, a consequence of the hampered breakdown of intrahepatic TG associated to the PNPLA3 I148M protein. Interestingly, in a cohort study in the Danish general population ( $n=94,708)$, the I148M ( $r$ 738409) was not causally associated with protection against ischemic heart disease (OR: $0.95 ; 95 \% \mathrm{Cl} 0.86-1.04)$, although ischemic heart disease increased stepwise with increasing liver fat content (OR: $2.41 ; 95 \% \mathrm{Cl} 1.28-4.51)^{61}$. However, it is worth mentioning that conflicting results have been also reported in the few observational studies that assessed the association between PNPLA3 rs738409 SNP and evaluation of c-IMT ${ }^{58}$.

The rs58542926 T-allele variant in the TM6SF2 gene was also found to be protective against CAD 60 and a systematic evaluation of coding variants in $>10,000$ Norwegians revealed that the TM6SF2 rs58542926 variation reduced total cholesterol and the incidence of myocardial infarction $(\mathrm{OR}=0.87 ; 95 \% \mathrm{Cl} 0.79-0.95){ }^{62}$. Contrasting data have been provided in the case of 
rs641738 T-allele (MBOAT7) and risk of CAD, i.e., a neutral effect ${ }^{60}$ or an increased risk of venous thromboembolism ${ }^{63}$. Finally, in 2017, Liu et al. tested the association of genotypes from the Human Exome Bead Chip with lipid levels and found that both TM6SF2 rs58542926 and PNPLA3 rs738409 variants were associated with lower lipid levels and a lower risk of CAD, but with an increased risk of steatosis and T2D ${ }^{37}$. Finally, the genetic variant $\mathrm{rs} 641738 \mathrm{C}>\mathrm{T}$ near MBOAT7, predisposing to raised hepatic fat, MAFLD and susceptibility to develop NASH, did not associate with CAD risk ${ }^{64}$.

To sum-up, variants which represent the best genetic predictors of NAFLD are associated with reduced CAD risk or at least do not predispose to CAD. The epidemiological association between fatty liver and cardiovascular damage may be mostly mediated by dyslipidemia and IR, both classical risk factors for atherosclerosis. To definitively state whether the genetics of NAFLD can affect CVD, other variations predisposing to NAFLD without affecting lipid secretion and plasma cholesterol and TG should be considered.

3.3 Epigenetic programmes in NAFLD. Nowadays, genetic variants associated with NAFLD account for only a minor fraction of the overall heritability. Missing information may be attributed to rare variants, to common variants that have not reached genome-wide significance, or to epigenetic modifications (in particular miRNAs) ${ }^{65,66}$.

An altered miRNA profile has been described in NAFLD and NASH, both in humans and experimental models ${ }^{67}$. Pirola et al explored the circulating miRNA signature associated with NAFLD revealing that, among 84 miRNAs analyzed, miR-122, miR-192, miR-19a/b, miR-125b and miR-375 were up-regulated in simple steatosis and, more so, the expression of miR-122, miR-192 and miR-375 in NASH, potentially distinguishing this condition from simple steatosis 68 . Differentially expressed miRNAs have been associated with hepatic fibrosis and progression to HCC. Cermelli et al. observed that circulating levels of miR-122, miR-34a and miR-16 were strongly correlated with liver enzymes, inflammatory activity and fibrosis score ${ }^{69}$. Expression of miR15 and miR16 is reduced in activated HSCs and, together with miR-34, they are also involved in the regulation of cell cycle progression, proliferation and hepatocarcinogenesis ${ }^{70}$.

Epigenetic changes may play a role in the fetal programming of liver fat ${ }^{71}$. Elevation of ALT was associated with HIF3A methylation in children with NAFLD, thus suggesting that 
epigenetic changes in the oxidative stress response predispose to fatty liver ${ }^{72}$. Maternal obesity and infant nutritional habits may be associated with methylation of the peroxisome proliferatoractivator receptor $\gamma$ coactivator-1 alpha (PGC1 $\alpha$ ) gene ${ }^{73}$, a key regulator of mitochondrial biogenesis and fatty acid oxidation in NAFLD ${ }^{74}$. Acetylation patterns also play a role. In utero exposure to maternal high-fat diet increased fetal H3K14 histone acetylation with a parallel decrease in SIRT1 expression and histone deacetylase activity, linked to an altered expression of genes mediated by SIRT1 and involved in fatty acid oxidation and lipogenesis ${ }^{75}$.

In patients with biopsy-proven NAFLD, the decreased expression of PGC1 $\alpha$ was inversely related with its promoter methylation. The latter was also positively related with plasma fasting insulin and HOMA-IR, thus suggesting that the IR phenotype and epigenetic changes are tightly intertwined with fatty liver development ${ }^{76}$. The protective mechanism driven by PGC1 $\alpha$ against hepatic steatosis and IR occurs by enhancing the IL-10 mediated anti-inflammatory response ${ }^{77}$. A novel epigenetic regulator of lipogenesis is hepatic Slug, a transcriptional factor which binds to the promoter of lipogenic genes fatty acid synthase (FASN), acetyl CoA Carboxylase 1 (ACC1), and Sterol regulatory element-binding transcription factor 1 (SREBP1C) ${ }^{77}$. Insulin raises the expression of Slug in primary hepatocytes, followed by binding to fatty acid synthase, thus stimulating the lipogenic program. Deletion of Slug inhibits lipogenesis and protects against NAFLD and obesity ${ }^{78}$. Alterations in the methylation signature of hepatic and peripheral bloodderived DNA, including major regulatory loci of metabolic inflammatory and fibrotic pathways, have been evaluated in NAFLD patients. Epigenome wide-association studies (EWAS) identified methylation changes in genes involved in liver function, cholesterol synthesis and steatosis development approximately explaining $10 \%$ of the interindividual variation ${ }^{79}$. The CpG island (CpG99) in the genomic region of PNPLA3 was hypermethylated in NAFLD patients and CpG26 was hypomethylated, both possibly contributing to fibrosis severity ${ }^{80}$.

Data on the role of long non-coding RNAs (Inc-RNAs) and other non-coding RNAs in NAFLD are still preliminary. The hepatic expression of Lnc1-8q22.2 ${ }^{81}$ was significantly raised in patients with NASH, whereas that of Inc-RNA1 (BLNC1) was elevated in the liver of obese mice with NAFLD ${ }^{82}$. Liver specific inactivation of Blnc1, required for the induction of lipogenic genes, abrogated high-fat-diet-induced steatosis, IR and protected mice against NASH.

This article is protected by copyright. All rights reserved 
3.4 Ethnic differences. Reasons for racial disparities in the prevalence of NAFLD are not fully understood. Genetic heritage across ethnic groups may be associated with the prevalence of the PNPLA3 at risk allele, occurring more frequently in Hispanic (49\%) followed by non Hispanic whites (23\%) and African American individuals (17\%) ${ }^{26}$. Moreover, population-based data have shown a clear ethnic footprint in fatty liver prevalence. This appears to be highest, both for NAFLD and NASH, in Hispanic individuals, intermediate in Whites and lowest in Blacks, whereas fibrosis does not appear to differ according to ethnicity ${ }^{83}$. Fatty liver appears to be increasing in Asian populations with an apparent more severe course ${ }^{84}$. Asian individuals seem to have a higher risk of fibrosis, whereas this risk is lower in African individuals compared to whites 85 .

3.5 Age and sex differences. These features have a definite influence on the likelihood of overall and disease specific mortality. Advancing age ${ }^{86}$ leads to substantial changes in liver structure with reduced blood flow and volume, together with reduced bile acid synthesis and alterations in cholesterol metabolism. Aging also leads to changes in body composition, including decreased muscle mass, raised abdominal adiposity and development of $I R^{87}$. Concerning sex-differences, as elsewhere extensively reviewed ${ }^{88}$, the overall prevalence of NAFLD is higher in men than in women, and becomes similar after the age of 50-60 years ${ }^{89}$. On this matter, although no specific studies have been designed to assess sex differences in NAFLD, a large meta-analysis comprising 62,239 individuals with NAFDL showed that, compared to men, women had a $19 \%$ lower risk of NAFLD with a relative risk of $0.81(95 \% \mathrm{Cl}$ 0.68-0.97), but once established, the risk of advanced fibrosis is higher in women especially after the age of $50{ }^{90}$. Interestingly, application of the MAFLD definition to a cohort of 756 Japanese with a previous diagnosis of NAFLD showed that MAFLD patients were more likely to be men ${ }^{15}$. In search of possible pathophysiological mechanisms explaining sex differences in NAFLD, it can be acknowledged the $\mathrm{X}$ chromosome dosage, significance of sex hormones, sex/gender-associated IR, as well as the distinct regional fat distribution and adipocyte biology ${ }^{89}$. Finally, considering that over 1,000 hepatic genes are differentially expressed between female and male livers, roles of peroxisome proliferator$\alpha$ activated receptor (PPAR $\alpha$ ), farnesoid $X$ receptor (FXR) and liver $X$ receptor (LXR) regulatory factors have to be acknowledged ${ }^{91}$.

This article is protected by copyright. All rights reserved 
3.6 Obesity. It can be classified as metabolically healthy and unhealthy, although individuals who are reported as having metabolically healthy obesity are often not truly healthy, but have fewer cardiometabolic abnormalities vs metabolically unhealthy individuals ${ }^{92}$. Overall, the metabolically unhealthy obese are at greater risk of T2D, CVD and all-cause mortality than those with metabolically healthy obesity ${ }^{93}$. Factors involved in the transition from metabolically healthy to metabolically unhealthy obesity are a prolonged excess adiposity, a decline in IR, a rise in fasting plasma glucose as well as the presence of NAFLD. This last condition worsens hepatic and systemic IR, favors atherogenic dyslipidemia and leads to the release of proinflammatory mediators ${ }^{94}$. A significant proportion of metabolically healthy obese display an altered fat distribution, confirming that a higher amount of visceral fat leads to a raised CV metabolic risk ${ }^{95}$. Visceral obesity is linked to enhanced inflammation and fibrosis, independent of IR and steatosis 96 .

A number of favorable adiposity genes have been recently identified and may be crucial in predicting fibrosis and cirrhosis risk in overweight/obese individuals ${ }^{97}$. In contrast to obesity associated NAFLD, in lean individuals (BMI $<25 \mathrm{~kg} / \mathrm{m}^{2}$ ) without "significant" alcohol intake, NAFLD occurs more frequently in Asian populations. In this ethnicity between 5 and $45 \%$ of patients with NAFLD are lean ${ }^{98}$, apparently with an accelerated disease progression.

3.7 Alcohol consumption. The threshold of alcohol intake in NAFLD patients is $30 \mathrm{~g} /$ day for men and $20 \mathrm{~g}$ for women, below which it does not induce steatosis or exert damaging effects on liver disease progression ${ }^{99}$. This general consensus has led to the recent debate on the safe limits on alcohol consumption in the setting of NAFLD ${ }^{100}$. This debate has led to the conclusion that alcohol intake has likely a straight dose-response toxicity, rather than a J-shaped association with liver disease and with synergistic damaging effects in the presence of the metabolic syndrome. This supports the ongoing change in nomenclature ${ }^{101,102}$, also improving the definition of the relationship with $\mathrm{CHD}^{5}$.

\section{NAFLD and risk of cardiovascular diseases}

A number of reports have conclusively established that NAFLD is an independent CV risk factor, considering in particular the similarity in risk profile between NAFLD and the metabolic syndrome 
6,103,104. As elsewhere reviewed ${ }^{105}$, NAFLD associates with increased risks of myocardial infarction, subclinical coronary or carotid atherosclerosis, as well as of valvular heart disease and the magnitude of risk correlates to the severity of NAFLD 106. Although no definite pathophysiological mechanisms explain this liaison, some hypotheses rely on hypertension, increased arterial stiffness ${ }^{107}$, hyperuricemia, a rise in the hepatic production of multiple prothrombogenic factors, increased oxidative stress and adipose tissue inflammation, as well as an altered adipokine profile ${ }^{108}$.

4.1 Lipid profile. The liver plays a central role in lipoprotein metabolism as it participates in the production and/or clearance of all classes of lipoprotein particles. As elsewhere reviewed ${ }^{109}$, alterations in hepatic lipid metabolism that lead to NAFLD also drive the development of atherogenic dyslipidemia.

Elevated triglyceride is an almost constant finding in patients with NAFLD. In the presence of steatosis, TG elevations are in particular associated with coronary plaques, both calcified and non-calcified, as well as with non-obstructive coronary stenoses ${ }^{110}$. Besides being correlated per se with calcified plaques independent of the presence of the metabolic syndrome in NAFLD patients ${ }^{111}$, higher TG and remnant cholesterol have been observationally and genetically associated with an increased risk of aortic valve stenosis ${ }^{112}$. Overall, the association between serum TG and hepatic steatosis is largely accounted for by a greater TG enrichment in VLDL particles ${ }^{113}$. On this matter, Boren et al have demonstrated by kinetic studies how $\mathrm{VLDL}_{1}-\mathrm{TG}$ production is $35 \%$ lower in homozygous TM6SF2 E167K carriers ${ }^{114}$. This finding provides a basis for understanding the lower $\mathrm{CV}$ risk associated with this mutation ${ }^{36}$, associated with a rise in hepatic lipid droplet TG content ${ }^{115}$ and predisposing to NAFLD ${ }^{116}$.

TG can be a crucial factor in the progression from NAFLD to NASH. VLDL synthesis is impaired in NASH, possibly as the result of lipid oxidative DNA damage, leading to dysfunctional VLDL synthesis and lipid outflow as key factors in the progression to NASH ${ }^{117}$. In the context of TG-rich lipoproteins, fasting remnant-cholesterol, another CV risk factor, is associated with the odds of NAFLD beyond traditional risk factors, such as adiposity and IR ${ }^{118}$. Fatty liver and elevated TG can be finally linked to apoprotein C3 (APOC3) variant alleles (C482T, T455C or both). Among healthy Asian Indian men, carriers of these APOC3 variants had a $30 \%$ increase in apoC3 
levels ${ }^{119}$, a $60 \%$ increase in fasting TGs and a $38 \%$ prevalence of fatty liver disease compared to wild type homozygotes. Similar conclusions were reached in a meta-analysis reporting that carriers of the genotype APOC3 rs2854116 had a $45 \%$ higher risk to develop NAFLD ${ }^{116}$. ApoC3 is also involved in glucose homeostasis, monocyte adhesion, activation of inflammatory pathways and modulation of the coagulation cascade ${ }^{120}$.

Besides the role played by TG-rich lipoproteins, altered levels of proatherogenic lipoprotein subclasses have been described, namely an increased percentage of small-dense (sd) LDL ${ }^{121}$. NAFLD patients may present low levels of larger $L L_{1}$ and increased smaller $L L_{3}$ and $L L_{4}$ particles leading to a more atherogenic profile.

The significant reduction of HDL-C in NAFLD appears to be associated with an impaired HDL cholesterol efflux capacity ${ }^{122}$. This last appears to have an independent negative correlation with c-IMT as well as with the presence of atherosclerotic plaques ${ }^{123}$, thus confirming the widely held view of a significant correlation between liver steatosis and CV risk. Significant apoAI reductions have been frequently reported in liver disease patients ${ }^{124}$ with a significant rise after resolution of fatty liver in patients after weight reduction ${ }^{125}$.

In the context of non-classical lipid biomarkers, the possible role played by both proprotein convertase subtilisin/kexin type 9 (PCSK9) (one of the key regulators of LDL-C) ${ }^{126}$ and PCSK7 is worth mentioning. We previously demonstrated that the PCSK7 rs236918 variant was associated with the severity of liver disease in biopsy-proven NAFLD patients, thus correlating with dyslipidemia and hepatic inflammation ${ }^{127}$. In the case of PCSK9, no definite conclusions have been reached. Although preclinical ${ }^{128}$ and retrospective studies found a positive association with the severity of fat accumulation ${ }^{129}$, genetic studies did not confirm this evidence ${ }^{130,131}$, leading to the conclusion that PCSK9 inhibition may not be linked to an increased risk of NAFLD.

4.2 Insulin resistance. Several data demonstrated that IR is a key player in the development of NAFLD and its progressive forms ${ }^{132}$. Indeed, IR correlates with the severity of liver fibrosis, the main determinant of NAFLD prognosis, and advanced fibrosis is often observed in T2D patients with NAFLD ${ }^{133}$. Moreover, genetic variants that dampen insulin receptor (InsR) signaling may favor fibrosis development in NAFLD ${ }^{134}$. IR also creates a pro-atherogenic environment for CVD development, i.e., by favoring dyslipidemia, hyperglycemia, activation of oxidative stress, 
endothelial dysfunction, and ectopic lipid accumulation ${ }^{135}$. What is clear today is that human genetic variations primarily increasing liver fat content do not have a direct effect on IR. It is the quality of fat rather than the quantity that causes IR ${ }^{136}$.

The molecular activation of lipogenesis in liver steatosis may be consequent to a raised glucose production, followed by conversion to fatty acids via pyruvate, entering the Krebs cycle 137. This synthesis, regulated by insulin, is mediated by the membrane bound transcription factor SREBP1C, not appropriately activated in the presence of IR. SREBP1C can activate the transcription of PPAR $\gamma$, a nuclear receptor required for normal adipocytes to achieve differentiation and involved in hepatic steatosis development. Deletion of PPAR $\gamma$ in different models leads to reduced steatosis independent of hyperinsulinemia and hyperglycemia ${ }^{138}$. Activation of PPAR $\gamma$, in contrast, leads to raised insulin secretion ${ }^{139}$ and may worsen steatosis, although clinical trials with the PPAR $\gamma$ agonist rosiglitazone have shown improved liver steatosis 140. Finally, AMP activated protein kinase (AMPK) stimulates fatty acid $\beta$ activation and lipogenesis ${ }^{141}$. Activation of AMPK occurs after the antidiabetic metformin, markedly reducing steatosis ${ }^{142}$ and liver size in man ${ }^{143}$.

4.3 Adipose tissue, ectopic fat accumulation and inflammation. Adipose tissue, beyond its role as a fat storage depot, is an endocrine organ, capable of producing and releasing biologically active proteins, named adipokines. These include leptin, adiponectin, tumor necrosis factor- $\alpha$, and interleukin (IL)-6. These molecules are not simply bystanders but exert different effects, which could improve or impair metabolic responses. Adipokines have been implicated in both NAFLD and CVD pathogenesis ${ }^{144}$. In particular, leptin and adiponectin may be appropriate biomarkers of NAFLD: low levels of adiponectin may predict independently the development of NAFLD, whereas leptin may be a significant predictor of NAFLD only in subjects with weight gain 145. In line with this evidence, we previously demonstrated that adiponectin levels are independently associated with the histological severity of NAFLD and the I148M PNPLA3 genotype may represent a genetic determinant of serum adiponectin ${ }^{146}$. In humans, adiponectin levels are positively correlated with HDL-C and inversely with TG and small-dense atherogenic LDL-C 147,148 . Leptin levels are instead raised in patients with vascular disease, including those 
with myocardial infarction and heart failure, as well as in those with coronary artery calcification and higher C-IMT ${ }^{149}$.

While obesity, particularly abdominal obesity, is an almost general accompanying marker of NAFLD, ectopic fats (pericardial fat, epicardial fat and pericoronary fat) are also of interest. All these can be measured by computed tomography (CT). Epicardial fat, in particular, is strongly associated with the risk of myocardial infarction 150 and the presence and progression of coronary artery calcification ${ }^{151}$. More recent findings indicate that epicardial fat thickness (EFT) is also strongly associated with obesity linked IR and also with liver steatosis ${ }^{152}$. EFT can be easily determined by echocardiography and is independently associated with left ventricular diastolic dysfunction and atherosclerotic lesions, as assessed by c-IMT. In addition, EFT can provide an early marker of CV injury in patients with IR ${ }^{153}$.

The accumulation of ectopic fat can thus be a marker of NAFLD and, while in humans it is best represented by EFT, in rodents it may be characterized by hepatocyte accumulation of neutral lipids forming lipid droplets (LDs) surrounded by proteins of which perilipin2 (PLIN2) is a major component ${ }^{154}$. PLIN2 appears to be a potentially protective factor against NAFLD ${ }^{155}$, since reduction of PLIN2 in monocytes occurs after bariatric surgery ${ }^{156}$. As a general consequence, obese patients with NAFLD treated with bariatric surgery show improved insulin sensitivity and reduced liver and monocyte fat accumulation ${ }^{6}$.

4.4 Inflammation. Chronic liver disease, as in the case of NAFLD, is fuelled by hepatic inflammation, being macrophages essential players in controlling this condition. Chronic fat overload induces liver cell death, resulting in the release of danger associated molecular patterns triggering macrophage activation ${ }^{157}$. During chronic liver injury, the increased recruitment of monocyte-derived macrophages and Kupffer cell populations leads to increased circulating levels of systemic inflammatory markers, including IL-1, IL-6 and subfamilies of IL-20 ${ }^{158}$. This process could worsen the CVD risk through endothelial dysfunction, altered vascular tone, raised plaque formation and clotting ${ }^{159}$.

4.5 Hypertension. Hypertension is one of the strongest risk factors for almost all different CVDs, including coronary and peripheral disease, left ventricular hypertrophy and valvular heart 
diseases, atrial fibrillation, stroke and renal failure ${ }^{160}$. Hypertension is definitely associated with IR and may be contributory or just associated with NAFLD ${ }^{161}$. The similarities between hypertension and NAFLD have led to the evaluation of potential treatments effective in both conditions. Lifestyle modification is an important strategy to manage NAFLD and hypertension. At the moment, however, only weight loss, avoidance of alcohol and some agents reducing IR such as metformin lead to blood pressure reduction ${ }^{162}$, thus improving both conditions. Proper strategies should be, however, the object of future investigations.

4.6 Microbiome. The interaction between gut and liver, the so-called "gut-liver axis" plays a critical role in the development and progression of NAFLD in both children and adults. An intact intestinal barrier is able to antagonize the translocation of bacterial products, while allowing active transport of nutrients across tight junctions. As reported in a recent meta-analysis, intestinal permeability appears to be raised in NAFLD patients, being associated with the degree of hepatic steatosis ${ }^{163}$. In this complex scenario, a crucial role is played by the intestinal microbiota, playing a major role in NAFLD. Recent data indicate that microbiome derived metabolites predict fibrosis and cirrhosis in NAFLD ${ }^{164}$. Gut flora and intestinal permeability regulate glucose, lipid and choline metabolism and have a clear impact on intestinal permeability 165. Increased circulating levels of bacterial products, i.e. lipopolysaccharides and other bioactive compounds, lead to the intrahepatic activation of proinflammatory cells and hepatocytes via stimulation of toll-like receptor-2 (TLR-2) ${ }^{166}$. In the attempt to find a link with CVD, one of the best candidates is represented by trimethylamine oxide (TMAO), derived from the metabolism of choline and carnitine by the gut microbiome ${ }^{167}$. TMAO has been extensively reported to be associated with the risk of fatal and non-fatal CV events. Its precursor trimethyllysine predicts near- and long-term CV events in patients with chest pain and acute coronary syndrome ${ }^{168}$. Conversely, although few studies have described changes in circulating levels of TMAO in NAFLD, it seems that TMAO associates with the severity of disease ${ }^{169}$ particularly in obese T2D patients with $\mathrm{NASH}^{170}$.

4.7 Epigenetics, NAFLD and CV risk. In a frame depicting NAFLD as a systemic disorder, miRNAs involved in the regulation of hepatic cholesterol and lipid metabolism, e.g., miR-122, miR-33a/b, 
and miR-29, may mediate the development of atherosclerosis and CVD ${ }^{171}$. In apoE ${ }^{-/}$mice, miR155 was significantly up-regulated in the aortas, and miR-155 deficiency inhibited the development of atherosclerosis. In miR155/-/apoE $\mathrm{E}^{-/-}$double $\mathrm{KO}$ mice, exposure to a high fat diet induced obesity, adipocyte hypertrophy and NAFLD ${ }^{172}$. In humans, miR-34 was upregulated in patients with CAD and more so in individuals with NAFLD ${ }^{172}$.

Finally, epigenetic changes and alterations in long non-coding RNAs (Inc-RNAs) expression are also involved in CVD. DNA methylation has been associated with atherosclerosis. In apoE ${ }^{-/}$ mice, DNA methylation precedes any histological sign of atherosclerosis and is correlated with dyslipidemia. Moreover, DNA methylation is associated with inflammatory markers such as IL-6, IL-8, IL1- $\beta$, C-reactive protein and vascular cell adhesion molecule-1 ${ }^{173}$. The expression of LncRNAs, known to be relevant in atherosclerosis, in plasma samples of 300 patients with CAD identified H19 and LIPCAR as independent predictors of CV damage ${ }^{174 .}$

5. Dietary approaches to NAFLD. Dietary intervention is the mainstay approach for the management of NAFLD, with a weight loss of $7-10 \%$ roughly considered as the goal. NAFLD patients can benefit by adhering to the Mediterranean low-carbohydrate diet, which can reduce hepatic steatosis and is recommended as the first-line dietary intervention in these patients ${ }^{175}$. Very recently the concept of green Mediterranean diet, enriched with specific green polyphenols, has been tested in the DIRECT PLUS (Dietary Intervention Randomized Controlled Trial Polyphenols Unprocessed) trial. Patients allocated to this approach had a larger intrahepatic fat loss (-2\% absolute change) compared to those allocated to the standard Mediterranean diet ($1 \%$ absolute change) or a healthy dietary approach (-0.7\% absolute change) ${ }^{176}$.

Very low energy diet (VLED) intake for periods of 6 days to 12 weeks can reduce liver volume in addition to visceral and subcutaneous adipose tissue and the reduction of liver volume appears to be directly related to a reduction in relative body weight ${ }^{177}$. As shown in an earlier study, a volume reduction of $20 \%$ was achieved after 12 weeks of VLED ${ }^{178}$. Interestingly, a significant reduction in liver fat, assessed by non-invasive quantitative magnetic resonance imaging (MRI), was observed after just 3 days of a low carbohydrate diet in normal volunteers. After 10 days, liver fat was reduced in the range between -1 to $-5 \%$ in all subjects ${ }^{179}$.

This article is protected by copyright. All rights reserved 
In view of the correlation between liver fat and total/animal protein intake, an attempt to reduce hepatic fat was carried out in the GRAANDIOOS (Improving Resilience With Whole Grain Wheat) study in 50 overweight subjects. Patients received either $98 \mathrm{~g} / \mathrm{d}$ of whole grain wheat or refined wheat and after 12 weeks there was a $49.1 \%$ rise of liver fat in the refined wheat group compared to stable levels in the whole grain wheat group ${ }^{180}$.

Several dietary components have been investigated for their association with the emergence of NAFLD. Among these are low intakes of PUFA and high intakes of saturated fat and cholesterol ${ }^{181}$. These findings have led to a number of randomized trials in patients with NAFLD by comparing dietary advice alone or with supplementations. In the case of $\omega-3$ PUFA a recent trial randomized 78 patients with NAFLD and hypertriglyceridemia to $4 \mathrm{~g} \omega-3$ fatty acids vs 200 $\mathrm{mg}$ fenofibrate or placebo for 12 weeks ${ }^{182}$. Regardless of the TG reduction, fenofibrate raised liver volume, whereas $\omega-3$ did not reduce liver fat, with no changes in liver enzymes and reduction of FGF21, independent of PNPLA3 polymorphism (I148M). This last finding supports the divergent effects of fenofibrate compared with $\omega-3$ on liver lipid accumulation and some potential benefits exerted by $\omega-3$ FA on hepatic metabolism ${ }^{183}$. Since the $\omega-6$ to $\omega-3$ ratio in the Western diet is on the average 15:1, van Name et al ${ }^{184}$ investigated in a series of obese youth with NAFLD, the impact of a low $\omega-6: \omega-3$ PUFA ratio (4:1) normo-caloric diet on hepatic fat amelioration. The treated youths had a $-25.8 \%$ reduction of liver fat with no changes in weight as well as a reduction in TG (-21.9\%) with a $-34 \%$ reduction in ALT and in oxidized TG metabolites. These changes were modulated by the PNPLA3 rs738409 genotype.

Low carbohydrate ketogenic diets have gained popularity in the treatment of obesity, T2D and also NAFLD ${ }^{185}$. This type of approach appears to be promising in the case of liver steatosis, where a hypocaloric ketogenic diet was found to reduce intrahepatic TG in 6 days by up to - $45 \%$ despite increased circulating FFA levels ${ }^{186}$. The highest reduction occurred in subjects homozygous for the PNPLA3-148 MM allele, i.e., -45 vs $+18 \%$ for the PNPLA3-148II group. This study, showing raised FFA in spite of reduction of liver fat, indicates a change of insulin action on liver FFA delivery, suggestive of impaired re-esterification of FA into complex lipids by altered mitochondrial fluxes. Quantification of liver mitochondrial metabolism in NAFLD patients under a ketogenic diet was very recently reported by Luukonen et al ${ }^{187}$. In these patients, isotopic 
infusions of the three precursors, glucose, hydroxybutyrate and lactate after 6 days of ketogenic diet reduced intrahepatic TGs by

$-31 \%$, again with raised FFA and reduced IR. FFAs appear to be partitioned toward ketogenesis, due to a reduction in serum insulin $(-53 \%)$ and of the citrate synthase flux. This latter was attributed to a raised mitochondrial redox state $(+167 \%)$ with a $-45 \%$ reduction of leptin and $21 \%$ of triiodothyronine. These findings may explain the reversal of NAFLD by ketogenic diet with altered hepatic mitochondrial fluxes and redox state promoting liver ketogenesis rather than synthesis of liver TG.

The PNPLA3 I148M variant represents a first and until now almost unique example of a genetic factor for which a clear interaction between the-gene and environment has been robustly demonstrated ${ }^{188}$. At the nutritional level, PNPLA3 expression is transcriptionally modulated by the activation of the SREBP1C/Liver X Receptor (LXR) pathway induced by hyperinsulinemia and by carbohydrate feeding ${ }^{189}$. In the double-blind placebo controlled WELCOME (Treatment of Non Alcoholic Fatty Liver Disease With n-3 Fatty Acids) trial, 103 adult patients with NAFLD were randomized to receive a supplementation of $\omega-3$ fatty acids (long chain PUFA), including docosahexaenoic (DHA) and eicosapentaenoic acid (EPA) or placebo for 15-18 months. PNPLA3 homozygous patients displayed an independent association with a decreased percentage of DHA tissue enrichment during the trial but not with changes in serum TG concentrations ${ }^{190}$. Consistently, in another randomized controlled trial, it was tested whether the PNPLA3 I148M variant is associated with the response to DHA (250 or $500 \mathrm{mg} / \mathrm{day}$ ) in 60 children with NAFLD for 24 months. This study demonstrated, however, that the $148 \mathrm{M}$ allele is associated with no beneficial effects of DHA supplementation on liver fat, showing a doubling of risk of severe steatosis at the end of the trial ${ }^{191}$. These findings were supported by Santoro et al. showing that PNPLA3 is involved in $\omega-3$ FA mobilization in the liver ${ }^{192}$. These may down-modulate SREBP1c expression and the PNPLA3 I148M variant is associated with lower DNL despite the substantial increase of hepatic fat content, thus explaining the lower response to DHA+EPA therapy in these patients.

5.1. Nutraceuticals. A number of nutraceuticals has been tested for their effects on NAFLD, e.g., silymarin, vitamin $E$, vitamin $D$, or resveratrol). Most of these have found support mainly from 
anecdotical reports with no clear evidence of benefit. As elsewhere reviewed ${ }^{193}$, the most widely used nutraceutical is silymarin, a powerful antioxidant agent extracted from milk thistle (Silybum marianum) with a specific liver tropism. Sylimarin has a low bioavailability, but, when given in combination with vitamin E, exerts a beneficial activity in NAFLD patients. Importantly, as in the case of resveratrol, a long-term appropriate dose supplementation has to be considered in order to achieve a clinical benefit in NAFLD patients ${ }^{194}$.

5.1.2 Probiotics. Obesity and nutrition may alter intestinal permeability producing a favorable micro-environment for bacterial overgrowth, mucosal inflammation and translocation of both invasive pathogens and harmful byproducts which influence liver fat composition and exacerbate pro-inflammatory and fibrotic processes ${ }^{195,196}$. Combination of probiotics, i.e. non pathogenic microorganisms with health benefits, with prebiotic fibers containing non digestible carbohydrates, modulates specific changes in the composition and activity of gastrointestinal microbiota, leading to the production of active synbiotics ${ }^{197}$. The use of a synbiotic yogurt (10 colony-forming unit (CFU) of Bifidobacterium animalis lactis as a probiotic and $1.5 \mathrm{~g}$ inulin as a prebiotic), has led to a significant reduction of liver enzymes, cholesterol, TGs and the grade of steatosis, in 102 patients with NAFLD and obesity ${ }^{198}$. Microbial-derived metabolite production, in particular short chain fatty acids can finally be important byproducts of prebiotic fibers ${ }^{199}$. In particular, butyrate can reduce inflammatory mediators and its role in NAFLD development is being currently investigated 200 .

6. Drug treatments. The numerous reports on dietary approaches for the management of liver steatosis/NAFLD do not match equivalent progress in drug treatment. Although up to now there is not a single drug specifically targeted to NAFLD, roughly 300 molecules are in various stages of development with this indication ${ }^{201}$.

Metformin has been thought for many years to exert beneficial effects on NAFLD through mechanisms involving an AMPK dependent improvement of hepatic glucose metabolism and increased uptake into muscle cells ${ }^{202}$. However, this mechanism has been recently challenged 203. Newer activities of metformin have been detected, in particular at the intestinal level. Reduced intestinal absorption, by way particularly of reduced endotoxin in portal plasma, leads 
to improved intestinal barrier function and markedly attenuates NAFLD induced by fat, fructose and cholesterol rich diets ${ }^{204}$. Metformin intake in humans is associated with a decrement in Bacteroides fragilis, a bacterial strain mediating barrier function ${ }^{205}$. Improved insulin sensitivity may be linked to an increased microbiomal prevalence of a beneficial bacterial strain, i.e., Akkermansia muciniphila, associated with loss of weight and improved liver fat and function in experimental models ${ }^{206}$. In diabetics given metformin, a high relative abundance of Akkermansia in intestinal microbiota with a consequent moderate loss of weight was reported ${ }^{207}$.

6.1 Agents acting on the PPAR system. While the PPAR $\alpha$ activator fenofibrate did not appear to provide any significant benefit on NAFLD ${ }^{182}$, because of its potential to raise liver volume and somewhat elevate liver lipid content, a beneficial activity can be exerted by the new agent Pemafibrate. It is $>2,000$-fold more selective for PPAR $\alpha$ vs either PPAR $\gamma$ or PPAR ${ }^{208}$. Preclinical studies in mice found that pemafibrate improved macrophage accumulation, ballooning degeneration of hepatocytes, and NAFLD without affecting TG accumulation in the liver ${ }^{209}$. This evidence has been confirmed in? NAFLD patients in whom pemafibrate improved markers of hepatic inflammation, function and fibrosis ${ }^{210}$. Moving to the CV risk, the PROMINENT (Pemafibrate to Reduce Cardiovascular Outcomes by Reducing Triglycerides in Patients with Diabetes) study will evaluate the efficacy of pemafibrate in reducing major adverse CV events in T2D patients with elevated TG. It is expected to be completed in $2024{ }^{211}$.

Pioglitazone has been extensively studied in the treatment of NASH ${ }^{212}$. By improving liver insulin sensitivity, pioglitazone can provide CV benefit, particularly in secondary prevention ${ }^{213}$. The earlier PPAR $\gamma$ activator rosiglitazone had been evaluated in patients with NASH in the FLIRT (Rosiglitazone for nonalcoholic steatohepatitis: one-year results of the randomized placebocontrolled Fatty Liver Improvement with Rosiglitazone Therapy) trial. One-year treatment (8 $\mathrm{mg} /$ day) led to a mean reduction of $47 \%$ in steatosis compared to placebo $(-16 \%)$ without significant improvement in histological parameters ${ }^{140}$. The initial FLIRT trial was followed by a 2year extension (FLIRT2 trial) without further significant improvement ${ }^{214}$. More convincing data have been provided by RCTs with pioglitazone, mainly in NASH. In patients with biopsy proven NASH and either prediabetes or T2D, pioglitazone (titrated to $45 \mathrm{mg} /$ day) in adjunction to a hypocaloric diet improved inflammation, ballooning, necrosis and steatosis compared to placebo

This article is protected by copyright. All rights reserved 
215. The PIVENS (Pioglitazone vs Vitamin E vs Placebo for Treatment of Non-Diabetic Patients With Nonalcoholic Steatohepatitis) trial, comparing pioglitazone (30 mg/day) vs vitamin E (800 IU/day) or placebo gave evidence that more patients on vitamin $E$ achieved the primary composite endpoint, including improvement in the NASH activity score, while the difference between pioglitazone and placebo did not reach the prespecified goal. Although pioglitazione did not reduce fibrosis, the histological resolution of NASH was found in $47 \%$ of patients compared to $21 \%$ on placebo $(p=0.001)^{216}$. The side effects of pioglitazone remain of concern, since it may cause weight gain, sodium/water retention and osteoporosis and potentially an increased risk of CV events ${ }^{217}$.

6.2 Newer antidiabetic agents. The sodium/glucose cotransporter-2 (SGLT2) inhibitors have provided a novel approach to diabetes. SGLT2 are expressed in the proximal renal tubules and are responsible for the majority of glucose reabsorption. SGLT2 inhibitors promote urinary glucose excretion and, in addition, reduce body weight and blood pressure with low risk of hypoglycemia ${ }^{218}$. These compounds appear to have an excellent profile for managing steatosis, as shown in animal models where the drugs reduced both liver TG accumulation and fibrosis ${ }^{219}$. After positive data in animal models ${ }^{220}$, in humans, treatment with SGLT2 inhibitors was shown to improve liver structure and function in patients with T2D ${ }^{221}$. SGLT-2 inhibitors reduce CV deaths and all-cause deaths compared to placebo (OR $0.77,95 \% \mathrm{Cl} 0.60-0.98$ and $\mathrm{OR} 0.67,95 \% \mathrm{Cl}$ $0.54-0.84)^{222}$.

Two new classes of antidiabetic agents have been tested in NAFLD. Agents acting on the glucagon-like peptide 1 (GLP-1) are further divided into GLP-1 receptor (GLP-1R) agonists and dipeptidylpeptidase-4 (DPP-4) inhibitors. GLP-1 is a naturally occurring gastrointestinal (GI) hormone secreted by L-cells of the distal small intestine, that regulate glucose in systemic and splanchnic vessels by stimulating glucose-dependent insulin secretion and inhibiting glucagon release ${ }^{223}$. GLP-1R agonists such as liraglutide, exenatide, dulaglutide and senaglutide seem to have a potential protective action on liver function. Seventy-two week treatment with semaglutide $(0.4 \mathrm{mg})$ resulted in a significantly higher percentage of patients with NASH resolution than placebo, $43 \%$ vs $33 \%$, respectively 224 . 
Agents acting by a similar mechanism as the GLP-1R agonists are the dideptidylpeptidase4 (DPP-4) inhibitors. DPP-4 is an enzyme breaking down bioactive enzymes, including the glucose dependent insulinotropic polypeptide (GIP) ${ }^{225}$. Inhibition reduces insulin secretion and suppresses glucagon. DPP-4 inhibitors were developed after the identification of the therapeutic activity of GLP1-R, in order to delay rapid inactivation and increase the incretin effect. The activity on NAFLD of DPP-4 inhibitors (sitagliptin, saxagliptin, linagliptin, alogliptin and vidagliptin) seems to be due to suppressed proinflammatory and profibrotic phenotypes of macrophages ${ }^{226}$. Although retrospective studies indicated reduced lipid abnormalities in patients with NASH or T2D, as well as improvement of liver enzymes ${ }^{227}$, more recent trials with sitagliptin did not provide clear statistically significant effects 228 .

6.3 Farnesoid $X$ receptor (FXR) agonist. FXR is a bile acid nuclear receptor playing a diversified role in lipoprotein and glucose metabolism, and also controlling hepatic fibrosis and inflammation ${ }^{229}$. A reduced expression of FXR raises the risk of developing NASH and HCC in mice ${ }^{230}$, a finding confirmed by the decreased FXR expression in patients with NAFLD ${ }^{231}$. The novel opening on the management of FXR deficiency or reduced expression was provided by obeticholic acid (OCA), a semisynthetic variant of the natural bile acid chenodeoxycholic acid ${ }^{232}$. OCA is approved for the treatment of primary biliary cholangitis and progressive autoimmune liver disease in patients with inadequate response to, or unable to tolerate, ursodeoxycholic acid 233. In NAFLD patients with T2D, OCA ( $25 \mathrm{mg} /$ day) raised insulin sensitivity by $28 \%$ and by $20.1 \%$ with the $50 \mathrm{mg} /$ day dose. A significant reduction in markers of liver fibrosis with a lower dose was also found ${ }^{232}$.

In the FLINT (Farnesoid X nuclear receptor ligand obeticholic acid for non-cirrhotic, nonalcoholic steatohepatitis) trial, enrolling 283 biopsy-proven NAFLD patients, 72-week treatment with OCA ( $25 \mathrm{mg} /$ day) led to a histological improvement in $45.4 \%$ of patients vs $21.1 \%$ in the placebo group ${ }^{234}$. More recently, Younossi et al 235 in the REGENERATE (Randomized Global Phase 3 Study to Evaluate the Impact on NASH With Fibrosis of Obeticholic Acid Treatment) study reported improvement in fibrosis and other key components of NASH upon 18 months (interim analysis) of treatment with OCA (clinical outcomes are expected). FXR agonism can lead to acute liver injury in rodent models of cholestasis. These findings could perhaps improve 
understanding of side effects of OCA, i.e., itching and hepatic decompensation in cirrhotic patients. However, acute liver injury has never been reported in the clinic ${ }^{236}$.

6.4 Ezetimibe. Ezetimibe, widely used for the treatment of hypercholesterolemia and CV risk reduction ${ }^{237}$ may be effective in treating NAFLD, especially in combination with fish oil 238 . Clinical studies with ezetimibe (without fish oil) have provided discordant results ${ }^{239}$. Ezetimibe inhibits the Nieman-Pick C1-Like 1 (NPC1L1) sterol receptor, a cellular protein expressed in enterocytes and in the liver. It regulates dietary and biliary cholesterol absorption ${ }^{240}$. A stimulated expression of cholesterol efflux transporters was very recently described in rats on fish oil. Those receiving $10 \%$ fish oil $+0.005 \%$ ezetimibe showed no increase in fecal cholesterol but a dramatic rise in the expression of ATP-binding cassette (ABC)G5/ABCG8 gene expression in the liver. Raised gene expression was associated with an $84 \%$ and $86 \%$ reduction of hepatic TG and cholesterol, respectively. ABCG5/ABCG8 form a heterodimeric complex responsible for biliary and transintestinal secretion of cholesterol and dietary sterols ${ }^{241}$. Finally, the recent finding that the overexpression of NPC1L1 in the liver exacerbates NAFLD, a pathological finding rescued by ezetimibe, could be the leverage to study patients with higher levels of NPC1L1 ${ }^{242}$.

7. Conclusions. Striking evidence from epidemiological and observational studies gives an indisputable link between NAFLD and a raised risk of CVD, whereas mixed results were reported in carriers of allelic variants causally related to NAFLD development (e.g., PNPLA3, TM6SF2, MBOAT7, GCKR and 17 $\beta$-HSD13). However, these associations should be reconsidered following the proposal to change diagnostic criteria, in order to identify MAFLD patients with fatty liver disease who are at high risk of disease progression or have a greater risk of CVD. Indeed, besides embracing metabolic abnormalities, MAFLD is also comprehensive of inflammatory markers, i.e., hsCRP $\geq 2 \mathrm{mg} / \mathrm{L}$, a well-known CVD risk factor ${ }^{243}$. Finally, in the present lack of a targeted pharmacological approach, the MAFLD definition narrowing this disease to a metabolic disorder could encourage drug repurposing, e.g., SGLT2 inhibitors or new selective PPAR $\alpha$ agonists.

Conflict of Interest. The Authors have no conflict of interest relative to the content of this review article.

This article is protected by copyright. All rights reserved 
Funding: Cariplo Foundation to MR (2018-0511); Fondazione Carlo Sirtori.

This article is protected by copyright. All rights reserved 


\section{Reference}

1. Dongiovanni P, Ruscica M. Non-alcoholic fatty liver disease and cardiovascular disease: A still debated liaison. Eur J Prev Cardiol. 2020;27(10):1056-1058.

2. Younossi ZM, Koenig AB, Abdelatif D, Fazel Y, Henry L, Wymer M. Global epidemiology of nonalcoholic fatty liver disease-Meta-analytic assessment of prevalence, incidence, and outcomes. Hepatology. 2016;64(1):73-84.

3. Younossi Z, Anstee QM, Marietti M, et al. Global burden of NAFLD and NASH: trends, predictions, risk factors and prevention. Nat Rev Gastroenterol Hepatol. 2018;15(1):11-20.

4. Targher G, Corey KE, Byrne CD. NAFLD, and cardiovascular and cardiac diseases: Factors influencing risk, prediction and treatment. Diabetes Metab. 2020:101215.

5. Targher G, Day CP, Bonora E. Risk of cardiovascular disease in patients with nonalcoholic fatty liver disease. N Engl J Med. 2010;363(14):1341-1350.

6. Targher G, Byrne CD, Lonardo A, Zoppini G, Barbui C. Non-alcoholic fatty liver disease and risk of incident cardiovascular disease: A meta-analysis. $J$ Hepatol. 2016;65(3):589-600.

7. Zhou YY, Zhou XD, Wu SJ, et al. Nonalcoholic fatty liver disease contributes to subclinical atherosclerosis: A systematic review and meta-analysis. Hepatol Commun. 2018;2(4):376-392.

8. Dufour JF. Time to Abandon NASH? Hepatology. 2016;63(1):9-10.

9. Fouad Y, Waked I, Bollipo S, Gomaa A, Ajlouni Y, Attia D. What's in a name? Renaming 'NAFLD' to 'MAFLD'. Liver Int. 2020;40(6):1254-1261.

10. Rinella ME. Nonalcoholic fatty liver disease: a systematic review. JAMA. 2015;313(22):2263-2273.

11. Shiha G, Korenjak M, Eskridge W, et al. Redefining fatty liver disease: an international patient perspective. Lancet Gastroenterol Hepatol. 2020.

12. Eslam M, Sanyal AJ, George J, International Consensus P. MAFLD: A Consensus-Driven Proposed Nomenclature for Metabolic Associated Fatty Liver Disease. Gastroenterology. 2020;158(7):1999-2014 e1991. 
13. Wu Y, Kumar R, Huang J, Wang M, Zhu Y, Lin S. FIB-4 cut-off should be re-evaluated in patients with metabolic associated fatty liver disease (MAFLD). J Hepatol. 2021;74(1):247-248.

14. Eslam M, Newsome PN, Sarin SK, et al. A new definition for metabolic dysfunctionassociated fatty liver disease: An international expert consensus statement. J Hepatol. 2020;73(1):202-209.

15. Yamamura S, Eslam M, Kawaguchi T, et al. MAFLD identifies patients with significant hepatic fibrosis better than NAFLD. Liver Int. 2020.

16. Lin S, Huang J, Wang M, et al. Comparison of MAFLD and NAFLD diagnostic criteria in real world. Liver Int. 2020;40(9):2082-2089.

17. Targher G. Concordance between MAFLD and NAFLD diagnostic criteria in 'realworld' data. Liver Int. 2020;40(11):2879-2880.

18. Dongiovanni P, Anstee QM, Valenti L. Genetic predisposition in NAFLD and NASH: impact on severity of liver disease and response to treatment. Curr Pharm Des. 2013;19(29):5219-5238.

19. Schwimmer JB, Celedon MA, Lavine JE, et al. Heritability of nonalcoholic fatty liver disease. Gastroenterology. 2009;136(5):1585-1592.

20. Makkonen J, Pietiläinen KH, Rissanen A, Kaprio J, Yki-Järvinen H. Genetic factors contribute to variation in serum alanine aminotransferase activity independent of obesity and alcohol: a study in monozygotic and dizygotic twins. J Hepatol. 2009;50(5):1035-1042.

21. Loomba R, Schork N, Chen $\mathrm{CH}$, et al. Heritability of Hepatic Fibrosis and Steatosis Based on a Prospective Twin Study. Gastroenterology. 2015;149(7):1784-1793.

22. Tarnoki AD, Tarnoki DL, Bata P, et al. Heritability of non-alcoholic fatty liver disease and association with abnormal vascular parameters: a twin study. Liver Int. 2012;32(8):1287-1293.

23. Dongiovanni P, Stender S, Pietrelli A, et al. Causal relationship of hepatic fat with liver damage and insulin resistance in nonalcoholic fatty liver. J Intern Med. 2018;283(4):356-370.

24. Dongiovanni P, Donati B, Fares R, et al. PNPLA3 I148M polymorphism and progressive liver disease. World J Gastroenterol. 2013;19(41):6969-6978. 
25. Valenti L, Al-Serri A, Daly AK, et al. Homozygosity for the patatin-like phospholipase3/adiponutrin I148M polymorphism influences liver fibrosis in patients with nonalcoholic fatty liver disease. Hepatology. 2010;51(4):1209-1217.

26. Romeo S, Kozlitina J, Xing C, et al. Genetic variation in PNPLA3 confers susceptibility to nonalcoholic fatty liver disease. Nat Genet. 2008;40(12):1461-1465.

27. Pingitore P, Dongiovanni P, Motta BM, et al. PNPLA3 overexpression results in reduction of proteins predisposing to fibrosis. Hum Mol Genet. 2016;25(23):52125222.

28. BasuRay S, Wang Y, Smagris E, Cohen JC, Hobbs HH. Accumulation of PNPLA3 on lipid droplets is the basis of associated hepatic steatosis. Proc Natl Acad Sci U SA. 2019;116(19):9521-9526.

29. Negoita F, Blomdahl J, Wasserstrom S, et al. PNPLA3 variant M148 causes resistance to starvation-mediated lipid droplet autophagy in human hepatocytes. J Cell Biochem. 2019;120(1):343-356.

30. Luukkonen PK, Nick A, Hölttä-Vuori M, et al. Human PNPLA3-I148M variant increases hepatic retention of polyunsaturated fatty acids. JCI Insight. 2019;4(16).

31. Franko A, Merkel D, Kovarova M, et al. Dissociation of Fatty Liver and Insulin Resistance in I148M PNPLA3 Carriers: Differences in Diacylglycerol (DAG) FA18:1 Lipid Species as a Possible Explanation. Nutrients. 2018;10(9).

32. Luukkonen PK, Zhou Y, Sädevirta S, et al. Hepatic ceramides dissociate steatosis and insulin resistance in patients with non-alcoholic fatty liver disease. J Hepatol. 2016;64(5):1167-1175.

33. Carpino G, Pastori D, Baratta F, et al. PNPLA3 variant and portal/periportal histological pattern in patients with biopsy-proven non-alcoholic fatty liver disease: a possible role for oxidative stress. Sci Rep. 2017;7(1):15756.

34. Kozlitina J, Smagris E, Stender S, et al. Exome-wide association study identifies a TM6SF2 variant that confers susceptibility to nonalcoholic fatty liver disease. Nat Genet. 2014;46(4):352-356.

35. Goffredo M, Caprio S, Feldstein AE, et al. Role of TM6SF2 rs58542926 in the pathogenesis of nonalcoholic pediatric fatty liver disease: A multiethnic study. Hepatology. 2016;63(1):117-125. 
36. Dongiovanni P, Petta S, Maglio C, et al. Transmembrane 6 superfamily member 2 gene variant disentangles nonalcoholic steatohepatitis from cardiovascular disease. Hepatology. 2015;61(2):506-514.

37. Liu DJ, Peloso GM, Yu H, et al. Exome-wide association study of plasma lipids in $>300,000$ individuals. Nat Genet. 2017;49(12):1758-1766.

38. Pirola CJ, Sookoian S. The dual and opposite role of the TM6SF2-rs58542926 variant in protecting against cardiovascular disease and conferring risk for nonalcoholic fatty liver: A meta-analysis. Hepatology. 2015;62(6):1742-1756.

39. Luukkonen PK, Zhou Y, Nidhina Haridas PA, et al. Impaired hepatic lipid synthesis from polyunsaturated fatty acids in TM6SF2 E167K variant carriers with NAFLD. $J$ Hepatol. 2017;67(1):128-136.

40. Buch S, Stickel F, Trépo E, et al. A genome-wide association study confirms PNPLA3 and identifies TM6SF2 and MBOAT7 as risk loci for alcohol-related cirrhosis. Nat Genet. 2015;47(12):1443-1448.

41. Mancina RM, Dongiovanni P, Petta S, et al. The MBOAT7-TMC4 Variant rs641738 Increases Risk of Nonalcoholic Fatty Liver Disease in Individuals of European Descent. Gastroenterology. 2016;150(5):1219-1230.e1216.

42. Di Sessa A, Umano GR, Cirillo G, et al. The Membrane-bound O-Acyltransferase7 rs641738 Variant in Pediatric Nonalcoholic Fatty Liver Disease. J Pediatr Gastroenterol Nutr. 2018;67(1):69-74.

43. Krawczyk M, Rau M, Schattenberg JM, et al. Combined effects of the PNPLA3 rs738409, TM6SF2 rs58542926, and MBOAT7 rs641738 variants on NAFLD severity: a multicenter biopsy-based study. J Lipid Res. 2017;58(1):247-255.

44. Meroni M, Dongiovanni P, Longo $M$, et al. Mboat7 down-regulation by hyperinsulinemia induces fat accumulation in hepatocytes. EBioMedicine. 2020;52:102658.

45. Luukkonen PK, Zhou Y, Hyötyläinen T, et al. The MBOAT7 variant rs641738 alters hepatic phosphatidylinositols and increases severity of non-alcoholic fatty liver disease in humans. J Hepatol. 2016;65(6):1263-1265.

46. Meroni M, Longo M, Fracanzani AL, Dongiovanni P. MBOAT7 down-regulation by genetic and environmental factors predisposes to MAFLD. EBioMedicine. 2020;57:102866. 
47. Anstee QM, Darlay R, Cockell S, et al. Genome-wide association study of non-alcoholic fatty liver and steatohepatitis in a histologically characterised cohort( 23 ). J Hepatol. 2020;73(3):505-515.

48. Tan HL, Zain SM, Mohamed R, et al. Association of glucokinase regulatory gene polymorphisms with risk and severity of non-alcoholic fatty liver disease: an interaction study with adiponutrin gene. J Gastroenterol. 2014;49(6):1056-1064.

49. Kozian DH, Barthel A, Cousin E, et al. Glucokinase-activating GCKR polymorphisms increase plasma levels of triglycerides and free fatty acids, but do not elevate cardiovascular risk in the Ludwigshafen Risk and Cardiovascular Health Study. Horm Metab Res. 2010;42(7):502-506.

50. Abul-Husn NS, Cheng X, Li AH, et al. A Protein-Truncating HSD17B13 Variant and Protection from Chronic Liver Disease. N Engl J Med. 2018;378(12):1096-1106.

51. Su W, Wang Y, Jia X, et al. Comparative proteomic study reveals $17 \beta-H S D 13$ as a pathogenic protein in nonalcoholic fatty liver disease. Proc Natl Acad Sci U S A. 2014;111(31):11437-11442.

52. Adam M, Heikelä H, Sobolewski C, et al. Hydroxysteroid (17 $\beta$ ) dehydrogenase 13 deficiency triggers hepatic steatosis and inflammation in mice. Faseb $j$. 2018;32(6):3434-3447.

53. Luukkonen PK, Tukiainen T, Juuti A, et al. Hydroxysteroid 17- $\beta$ dehydrogenase 13 variant increases phospholipids and protects against fibrosis in nonalcoholic fatty liver disease. JCI Insight. 2020;5(5).

54. Gellert-Kristensen H, Nordestgaard BG, Tybjaerg-Hansen A, Stender S. High Risk of Fatty Liver Disease Amplifies the Alanine Transaminase-Lowering Effect of a HSD17B13 Variant. Hepatology. 2020;71(1):56-66.

55. Yang J, Trépo E, Nahon P, et al. A 17-Beta-Hydroxysteroid Dehydrogenase 13 Variant Protects From Hepatocellular Carcinoma Development in Alcoholic Liver Disease. Hepatology. 2019;70(1):231-240.

56. Eslam M, George J. Genetic contributions to NAFLD: leveraging shared genetics to uncover systems biology. Nat Rev Gastroenterol Hepatol. 2020;17(1):40-52. 
57. Speliotes EK, Yerges-Armstrong LM, Wu J, et al. Genome-wide association analysis identifies variants associated with nonalcoholic fatty liver disease that have distinct effects on metabolic traits. PLoS Genet. 2011;7(3):e1001324.

58. Lonardo A, Ballestri S, Targher G. "Not all forms of NAFLD were created equal". Do metabolic syndrome-related NAFLD and PNPLA3-related NAFLD exert a variable impact on the risk of early carotid atherosclerosis? Atherosclerosis. 2017;257:253255.

59. Mahajan A, Wessel J, Willems SM, et al. Refining the accuracy of validated target identification through coding variant fine-mapping in type 2 diabetes. Nat Genet. 2018;50(4):559-571.

60. Simons N, Isaacs A, Koek GH, Kuč S, Schaper NC, Brouwers M. PNPLA3, TM6SF2, and MBOAT7 Genotypes and Coronary Artery Disease. Gastroenterology. 2017;152(4):912-913.

61. Lauridsen BK, Stender S, Kristensen TS, et al. Liver fat content, non-alcoholic fatty liver disease, and ischaemic heart disease: Mendelian randomization and metaanalysis of 279013 individuals. Eur Heart J. 2018;39(5):385-393.

62. Holmen OL, Zhang H, Fan Y, et al. Systematic evaluation of coding variation identifies a candidate causal variant in TM6SF2 influencing total cholesterol and myocardial infarction risk. Nat Genet. 2014;46(4):345-351.

63. Tabassum R, Ramo JT, Ripatti P, et al. Genetic architecture of human plasma lipidome and its link to cardiovascular disease. Nat Commun. 2019;10(1):4329.

64. Ismaiel A, Dumitrascu DL. Genetic predisposition in metabolic-dysfunction-associated fatty liver disease and cardiovascular outcomes-Systematic review. Eur J Clin Invest. 2020;50(10):e13331.

65. Dongiovanni P, Meroni M, Longo M, Fargion S, Fracanzani AL. miRNA Signature in NAFLD: A Turning Point for a Non-Invasive Diagnosis. Int J Mol Sci. 2018;19(12).

66. Zhang X, Asllanaj E, Amiri M, et al. Deciphering the role of epigenetic modifications in fatty liver disease: A systematic review. Eur J Clin Invest. 2020:e13479.

67. Cheung O, Puri P, Eicken C, et al. Nonalcoholic steatohepatitis is associated with altered hepatic MicroRNA expression. Hepatology. 2008;48(6):1810-1820. 
68. Pirola CJ, Fernández Gianotti T, Castaño GO, et al. Circulating microRNA signature in non-alcoholic fatty liver disease: from serum non-coding RNAs to liver histology and disease pathogenesis. Gut. 2015;64(5):800-812.

69. Cermelli S, Ruggieri A, Marrero JA, Ioannou GN, Beretta L. Circulating microRNAs in patients with chronic hepatitis $\mathrm{C}$ and non-alcoholic fatty liver disease. PLoS One. 2011;6(8):e23937.

70. Otsuka M, Kishikawa $\mathrm{T}$, Yoshikawa $\mathrm{T}$, et al. The role of microRNAs in hepatocarcinogenesis: current knowledge and future prospects. J Gastroenterol. 2014;49(2):173-184.

71. Baker PR, 2nd, Friedman JE. Mitochondrial role in the neonatal predisposition to developing nonalcoholic fatty liver disease. J Clin Invest. 2018;128(9):3692-3703.

72. Wang S, Song J, Yang Y, Zhang Y, Wang H, Ma J. HIF3A DNA Methylation Is Associated with Childhood Obesity and ALT. PLoS One. 2015;10(12):e0145944.

73. Gemma C, Sookoian S, Alvarinas J, et al. Maternal pregestational BMI is associated with methylation of the PPARGC1A promoter in newborns. Obesity (Silver Spring). 2009;17(5):1032-1039.

74. Longo M, Meroni M, Paolini E, Macchi C, Dongiovanni P. Mitochondrial dynamics and nonalcoholic fatty liver disease (NAFLD): new perspectives for a fairy-tale ending? Metabolism. 2021;117:154708.

75. Suter MA, Chen A, Burdine MS, et al. A maternal high-fat diet modulates fetal SIRT1 histone and protein deacetylase activity in nonhuman primates. Faseb j. 2012;26(12):5106-5114.

76. Sookoian S, Rosselli MS, Gemma C, et al. Epigenetic regulation of insulin resistance in nonalcoholic fatty liver disease: impact of liver methylation of the peroxisome proliferator-activated receptor $\gamma$ coactivator $1 \alpha$ promoter. Hepatology. 2010;52(6):1992-2000.

77. Wan X, Zhu X, Wang H, et al. PGC1alpha protects against hepatic steatosis and insulin resistance via enhancing IL10-mediated anti-inflammatory response. FASEB J. 2020.

78. Liu Y, Lin H, Jiang L, et al. Hepatic Slug epigenetically promotes liver lipogenesis, fatty liver disease, and type 2 diabetes. J Clin Invest. 2020;130(6):2992-3004. 
79. Ma J, Nano J, Ding J, et al. A Peripheral Blood DNA Methylation Signature of Hepatic Fat Reveals a Potential Causal Pathway for Nonalcoholic Fatty Liver Disease. Diabetes. 2019;68(5):1073-1083.

80. Kitamoto T, Kitamoto A, Ogawa Y, et al. Targeted-bisulfite sequence analysis of the methylation of $\mathrm{CpG}$ islands in genes encoding PNPLA3, SAMM50, and PARVB of patients with non-alcoholic fatty liver disease. J Hepatol. 2015;63(2):494-502.

81. Atanasovska B, Rensen SS, van der Sijde MR, et al. A liver-specific long noncoding RNA with a role in cell viability is elevated in human nonalcoholic steatohepatitis. Hepatology. 2017;66(3):794-808.

82. Zhao XY, Xiong X, Liu T, et al. Long noncoding RNA licensing of obesity-linked hepatic lipogenesis and NAFLD pathogenesis. Nat Commun. 2018;9(1):2986.

83. Rich NE, Oji S, Mufti AR, et al. Racial and Ethnic Disparities in Nonalcoholic Fatty Liver Disease Prevalence, Severity, and Outcomes in the United States: A Systematic Review and Meta-analysis. Clin Gastroenterol Hepatol. 2018;16(2):198-210 e192.

84. Weston SR, Leyden W, Murphy R, et al. Racial and ethnic distribution of nonalcoholic fatty liver in persons with newly diagnosed chronic liver disease. Hepatology. 2005;41(2):372-379.

85. Mohanty SR, Troy TN, Huo D, O'Brien BL, Jensen DM, Hart J. Influence of ethnicity on histological differences in non-alcoholic fatty liver disease. J Hepatol. 2009;50(4):797804.

86. Angulo P, Kleiner DE, Dam-Larsen S, et al. Liver Fibrosis, but No Other Histologic Features, Is Associated With Long-term Outcomes of Patients With Nonalcoholic Fatty Liver Disease. Gastroenterology. 2015;149(2):389-397.e310.

87. Assuncao N, Sudo FK, Drummond C, de Felice FG, Mattos P. Metabolic Syndrome and cognitive decline in the elderly: A systematic review. PLoS One. 2018;13(3):e0194990.

88. Lonardo A, Suzuki A. Sexual Dimorphism of NAFLD in Adults. Focus on Clinical Aspects and Implications for Practice and Translational Research. J Clin Med. 2020;9(5). 
89. Lonardo A, Nascimbeni F, Ballestri S, et al. Sex Differences in Nonalcoholic Fatty Liver Disease: State of the Art and Identification of Research Gaps. Hepatology. 2019;70(4):1457-1469.

90. Balakrishnan M, Patel P, Dunn-Valadez S, et al. Women Have a Lower Risk of Nonalcoholic Fatty Liver Disease but a Higher Risk of Progression vs Men: A Systematic Review and Meta-analysis. Clin Gastroenterol Hepatol. 2021;19(1):61-71 e15.

91. Cvitanovic Tomas T, Urlep Z, Moskon M, Mraz M, Rozman D. LiverSex Computational Model: Sexual Aspects in Hepatic Metabolism and Abnormalities. Front Physiol. 2018;9:360.

92. Smith GI, Mittendorfer B, Klein S. Metabolically healthy obesity: facts and fantasies. J Clin Invest. 2019;129(10):3978-3989.

93. Mongraw-Chaffin M, Foster MC, Anderson CAM, et al. Metabolically Healthy Obesity, Transition to Metabolic Syndrome, and Cardiovascular Risk. J Am Coll Cardiol. 2018;71(17):1857-1865.

94. Lonardo A, Mantovani A, Lugari S, Targher G. Epidemiology and pathophysiology of the association between NAFLD and metabolically healthy or metabolically unhealthy obesity. Ann Hepatol. 2020;19(4):359-366.

95. van der Poorten D, Milner KL, Hui J, et al. Visceral fat: a key mediator of steatohepatitis in metabolic liver disease. Hepatology. 2008;48(2):449-457.

96. Zeyda M, Stulnig TM. Obesity, inflammation, and insulin resistance--a mini-review. Gerontology. 2009;55(4):379-386.

97. Ji Y, Yiorkas AM, Frau F, et al. Genome-Wide and Abdominal MRI Data Provide Evidence That a Genetically Determined Favorable Adiposity Phenotype Is Characterized by Lower Ectopic Liver Fat and Lower Risk of Type 2 Diabetes, Heart Disease, and Hypertension. Diabetes. 2019;68(1):207-219.

98. Kim D, Kim WR. Nonobese Fatty Liver Disease. Clin Gastroenterol Hepatol. 2017;15(4):474-485.

99. Chalasani N, Younossi Z, Lavine JE, et al. The diagnosis and management of nonalcoholic fatty liver disease: Practice guidance from the American Association for the Study of Liver Diseases. Hepatology. 2018;67(1):328-357. 
100. Meltzer-Brody S, Colquhoun H, Riesenberg R, et al. Brexanolone injection in postpartum depression: two multicentre, double-blind, randomised, placebo-controlled, phase 3 trials. Lancet. 2018;392(10152):1058-1070.

101. Aberg F, Helenius-Hietala J, Puukka P, Farkkila M, Jula A. Interaction between alcohol consumption and metabolic syndrome in predicting severe liver disease in the general population. Hepatology. 2018;67(6):2141-2149.

102. Boyle M, Masson S, Anstee QM. The bidirectional impacts of alcohol consumption and the metabolic syndrome: Cofactors for progressive fatty liver disease. J Hepatol. 2018;68(2):251-267.

103. Diehl AM, Day C. Cause, Pathogenesis, and Treatment of Nonalcoholic Steatohepatitis. N Engl J Med. 2017;377(21):2063-2072.

104. Gill C, Vatcheva KP, Pan JJ, et al. Frequency of Nonalcoholic Fatty Liver Disease and Subclinical Atherosclerosis Among Young Mexican Americans. Am J Cardiol. 2017;119(11):1717-1722.

105. Targher G, Byrne CD, Tilg H. NAFLD and increased risk of cardiovascular disease: clinical associations, pathophysiological mechanisms and pharmacological implications. Gut. 2020;69(9):1691-1705.

106. Taylor RS, Taylor RJ, Bayliss S, et al. Association Between Fibrosis Stage and Outcomes of Patients With Nonalcoholic Fatty Liver Disease: A Systematic Review and Meta-Analysis. Gastroenterology. 2020;158(6):1611-1625 e1612.

107. Cicero AFG, Gitto S, Fogacci F, et al. Fatty liver index is associated to pulse wave velocity in healthy subjects: Data from the Brisighella Heart Study. Eur J Intern Med. 2018;53:29-33.

108. Lonardo A, Nascimbeni F, Mantovani A, Targher G. Hypertension, diabetes, atherosclerosis and NASH: Cause or consequence? J Hepatol. 2018;68(2):335-352.

109. Deprince A, Haas JT, Staels B. Dysregulated lipid metabolism links NAFLD to cardiovascular disease. Mol Metab. 2020;42:101092.

110. Cao YX, Zhang HW, Jin JL, et al. Prognostic utility of triglyceride-rich lipoproteinrelated markers in patients with coronary artery disease. J Lipid Res. 2020;61(9):1254-1262. 
111. Assy N, Djibre A, Farah R, Grosovski M, Marmor A. Presence of coronary plaques in patients with nonalcoholic fatty liver disease. Radiology. 2010;254(2):393-400.

112. Kaltoft M, Langsted A, Nordestgaard BG. Triglycerides and remnant cholesterol associated with risk of aortic valve stenosis: Mendelian randomization in the Copenhagen General Population Study. Eur Heart J. 2020;41(24):2288-2299.

113. Toledo FG, Sniderman AD, Kelley DE. Influence of hepatic steatosis (fatty liver) on severity and composition of dyslipidemia in type 2 diabetes. Diabetes Care. 2006;29(8):1845-1850.

114. Boren J, Adiels M, Bjornson E, et al. Effects of TM6SF2 E167K on hepatic lipid and very low-density lipoprotein metabolism in humans. JCI Insight. 2020.

115. Mahdessian H, Taxiarchis A, Popov S, et al. TM6SF2 is a regulator of liver fat metabolism influencing triglyceride secretion and hepatic lipid droplet content. Proc Natl Acad Sci U S A. 2014;111(24):8913-8918.

116. Tong M, Wang F. APOC3rs2854116, PNPLA3rs738409, and TM6SF2rs58542926 polymorphisms might influence predisposition of NAFLD: A meta-analysis. IUBMB Life. 2020;72(8):1757-1764.

117. Fujita K, Nozaki Y, Wada K, et al. Dysfunctional very-low-density lipoprotein synthesis and release is a key factor in nonalcoholic steatohepatitis pathogenesis. Hepatology. 2009;50(3):772-780.

118. Chin J, Mori TA, Adams LA, et al. Association between remnant lipoprotein cholesterol levels and non-alcoholic fatty liver disease in adolescents. JHEP Rep. 2020;2(6):100150.

119. Petersen KF, Dufour S, Hariri A, et al. Apolipoprotein C3 gene variants in nonalcoholic fatty liver disease. $N$ Engl J Med. 2010;362(12):1082-1089.

120. D'Erasmo L, Di Costanzo A, Gallo A, Bruckert E, Arca M. ApoCIII: A multifaceted protein in cardiometabolic disease. Metabolism. 2020;113:154395.

121. Siddiqui MS, Fuchs M, Idowu MO, et al. Severity of nonalcoholic fatty liver disease and progression to cirrhosis are associated with atherogenic lipoprotein profile. Clin Gastroenterol Hepatol. 2015;13(5):1000-1008 e1003. 
122. Fadaei R, Poustchi H, Meshkani R, Moradi N, Golmohammadi T, Merat S. Impaired HDL cholesterol efflux capacity in patients with non-alcoholic fatty liver disease is associated with subclinical atherosclerosis. Sci Rep. 2018;8(1):11691.

123. Khera AV, Cuchel $\mathrm{M}$, de la Llera-Moya $\mathrm{M}$, et al. Cholesterol efflux capacity, highdensity lipoprotein function, and atherosclerosis. N Engl J Med. 2011;364(2):127-135.

124. Floren CH, Franzen J, Albers JJ. Apolipoprotein A-I in liver disease. Scand J Gastroenterol. 1987;22(4):454-458.

125. Sung KC, Lee MY, Lee JY, et al. Resolution of fatty liver and weight loss: Independent associations with changes in serum lipids and apolipoproteins. Atherosclerosis. 2018;272:47-53.

126. Macchi C, Banach M, Corsini A, Sirtori CR, Ferri N, Ruscica M. Changes in circulating pro-protein convertase subtilisin/kexin type 9 levels - experimental and clinical approaches with lipid-lowering agents. Eur J Prev Cardiol. 2019;26(9):930-949.

127. Dongiovanni P, Meroni M, Baselli G, et al. PCSK7 gene variation bridges atherogenic dyslipidemia with hepatic inflammation in NAFLD patients. $J$ Lipid Res. 2019;60(6):1144-1153.

128. Lebeau PF, Byun JH, Platko K, et al. Pcsk9 knockout exacerbates diet-induced nonalcoholic steatohepatitis, fibrosis and liver injury in mice. JHEP Rep. 2019;1(6):418429.

129. Ruscica M, Ferri N, Macchi C, et al. Liver fat accumulation is associated with circulating PCSK9. Ann Med. 2016;48(5):384-391.

130. Grimaudo S, Bartesaghi S, Rametta R, et al. PCSK9 rs11591147 R46L loss-of-function variant protects against liver damage in individuals with NAFLD. Liver Int. 2020.

131. Rimbert A, Smati S, Dijk W, Le May C, Cariou B. Genetic Inhibition of PCSK9 and Liver Function. JAMA Cardiol. 2020.

132. Dongiovanni P, Rametta R, Meroni M, Valenti L. The role of insulin resistance in nonalcoholic steatohepatitis and liver disease development--a potential therapeutic target? Expert Rev Gastroenterol Hepatol. 2016;10(2):229-242.

133. McPherson S, Hardy T, Henderson E, Burt AD, Day CP, Anstee QM. Evidence of NAFLD progression from steatosis to fibrosing-steatohepatitis using paired biopsies: 
implications for prognosis and clinical management. J Hepatol. 2015;62(5):11481155.

134. Dongiovanni P, Valenti L, Rametta R, et al. Genetic variants regulating insulin receptor signalling are associated with the severity of liver damage in patients with nonalcoholic fatty liver disease. Gut. 2010;59(2):267-273.

135. Ormazabal V, Nair S, Elfeky O, Aguayo C, Salomon C, Zuniga FA. Association between insulin resistance and the development of cardiovascular disease. Cardiovasc Diabetol. 2018;17(1):122.

136. Tavaglione F, Targher G, Valenti L, Romeo S. Human and molecular genetics shed lights on fatty liver disease and diabetes conundrum. Endocrinol Diabetes Metab. 2020;3(4):e00179.

137. Browning JD, Horton JD. Molecular mediators of hepatic steatosis and liver injury. J Clin Invest. 2004;114(2):147-152.

138. Kim JB, Wright HM, Wright M, Spiegelman BM. ADD1/SREBP1 activates PPARgamma through the production of endogenous ligand. Proc Natl Acad Sci $U$ S A. 1998;95(8):4333-4337.

139. Kim HS, Hwang YC, Koo SH, et al. PPAR-gamma activation increases insulin secretion through the up-regulation of the free fatty acid receptor GPR40 in pancreatic betacells. PLoS One. 2013;8(1):e50128.

140. Ratziu V, Giral P, Jacqueminet S, et al. Rosiglitazone for nonalcoholic steatohepatitis: one-year results of the randomized placebo-controlled Fatty Liver Improvement with Rosiglitazone Therapy (FLIRT) Trial. Gastroenterology. 2008;135(1):100-110.

141. Hardie DG. Minireview: the AMP-activated protein kinase cascade: the key sensor of cellular energy status. Endocrinology. 2003;144(12):5179-5183.

142. Lin HZ, Yang SQ, Chuckaree C, Kuhajda F, Ronnet G, Diehl AM. Metformin reverses fatty liver disease in obese, leptin-deficient mice. Nat Med. 2000;6(9):998-1003.

143. Marchesini G, Brizi M, Bianchi G, Tomassetti S, Zoli M, Melchionda N. Metformin in non-alcoholic steatohepatitis. Lancet. 2001;358(9285):893-894.

144. Boutari C, Mantzoros CS. Adiponectin and leptin in the diagnosis and therapy of NAFLD. Metabolism. 2020;103:154028. 
145. Kim YS, Lee SH, Park SG, et al. Low levels of total and high-molecular-weight adiponectin may predict non-alcoholic fatty liver in Korean adults. Metabolism. 2020;103:154026.

146. Valenti L, Rametta R, Ruscica M, et al. The I148M PNPLA3 polymorphism influences serum adiponectin in patients with fatty liver and healthy controls. $B M C$ Gastroenterol. 2012;12:111.

147. Hulthe J, Hulten LM, Fagerberg B. Low adipocyte-derived plasma protein adiponectin concentrations are associated with the metabolic syndrome and small dense lowdensity lipoprotein particles: atherosclerosis and insulin resistance study. Metabolism. 2003;52(12):1612-1614.

148. Mantzoros CS, Li T, Manson JE, Meigs JB, Hu FB. Circulating adiponectin levels are associated with better glycemic control, more favorable lipid profile, and reduced inflammation in women with type 2 diabetes. J Clin Endocrinol Metab. 2005;90(8):4542-4548.

149. Ruscica M, Baragetti A, Catapano AL, Norata GD. Translating the biology of adipokines in atherosclerosis and cardiovascular diseases: Gaps and open questions. Nutr Metab Cardiovasc Dis. 2017;27(5):379-395.

150. Mahabadi AA, Berg MH, Lehmann N, et al. Association of epicardial fat with cardiovascular risk factors and incident myocardial infarction in the general population: the Heinz Nixdorf Recall Study. J Am Coll Cardiol. 2013;61(13):13881395.

151. Mahabadi AA, Lehmann N, Kalsch H, et al. Association of epicardial adipose tissue with progression of coronary artery calcification is more pronounced in the early phase of atherosclerosis: results from the Heinz Nixdorf recall study. JACC CardiovasC Imaging. 2014;7(9):909-916.

152. Baragetti A, Pisano G, Bertelli C, et al. Subclinical atherosclerosis is associated with Epicardial Fat Thickness and hepatic steatosis in the general population. Nutr Metab Cardiovasc Dis. 2016;26(2):141-153.

153. Gonzalez N, Moreno-Villegas Z, Gonzalez-Bris A, Egido J, Lorenzo O. Regulation of visceral and epicardial adipose tissue for preventing cardiovascular injuries associated to obesity and diabetes. Cardiovasc Diabetol. 2017;16(1):44. 
154. McIntosh AL, Senthivinayagam S, Moon KC, et al. Direct interaction of Plin2 with lipids on the surface of lipid droplets: a live cell FRET analysis. Am J Physiol Cell Physiol. 2012;303(7):C728-742.

155. Libby AE, Bales E, Orlicky DJ, McManaman JL. Perilipin-2 Deletion Impairs Hepatic Lipid Accumulation by Interfering with Sterol Regulatory Element-binding Protein (SREBP) Activation and Altering the Hepatic Lipidome. $J$ Biol Chem. 2016;291(46):24231-24246.

156. Angelini G, Castagneto Gissey L, Del Corpo G, et al. New insight into the mechanisms of ectopic fat deposition improvement after bariatric surgery. Sci Rep. 2019;9(1):17315.

157. Krenkel 0, Tacke F. Liver macrophages in tissue homeostasis and disease. Nat Rev Immunol. 2017;17(5):306-321.

158. He Y, Hwang S, Ahmed YA, et al. Immunopathobiology and therapeutic targets related to cytokines in liver diseases. Cell Mol Immunol. 2020.

159. Back M, Yurdagul A, Jr., Tabas I, Oorni K, Kovanen PT. Inflammation and its resolution in atherosclerosis: mediators and therapeutic opportunities. Nat Rev Cardiol. 2019;16(7):389-406.

160. Kjeldsen SE. Hypertension and cardiovascular risk: General aspects. Pharmacol Res. 2018;129:95-99.

161. Zhao YC, Zhao GJ, Chen Z, She ZG, Cai J, Li H. Nonalcoholic Fatty Liver Disease: An Emerging Driver of Hypertension. Hypertension. 2020;75(2):275-284.

162. Landin K, Tengborn L, Smith U. Treating insulin resistance in hypertension with metformin reduces both blood pressure and metabolic risk factors. J Intern Med. 1991;229(2):181-187.

163. De Munck TJI, Xu P, Verwijs HJA, et al. Intestinal permeability in human nonalcoholic fatty liver disease: A systematic review and meta-analysis. Liver Int. 2020.

164. Caussy C, Hsu C, Lo MT, et al. Link between gut-microbiome derived metabolite and shared gene-effects with hepatic steatosis and fibrosis in NAFLD. Hepatology. 2018;68(3):918-932.

165. Ilan Y. Leaky gut and the liver: a role for bacterial translocation in nonalcoholic steatohepatitis. World J Gastroenterol. 2012;18(21):2609-2618. 
166. Miura K, Yang L, van Rooijen N, Brenner DA, Ohnishi H, Seki E. Toll-like receptor 2 and palmitic acid cooperatively contribute to the development of nonalcoholic steatohepatitis through inflammasome activation in mice. Hepatology. 2013;57(2):577-589.

167. Tang WH, Hazen SL. The contributory role of gut microbiota in cardiovascular disease. J Clin Invest. 2014;124(10):4204-4211.

168. Li XS, Obeid S, Wang Z, et al. Trimethyllysine, a trimethylamine N-oxide precursor, provides near- and long-term prognostic value in patients presenting with acute coronary syndromes. Eur Heart J. 2019;40(32):2700-2709.

169. Chen YM, Liu Y, Zhou RF, et al. Associations of gut-flora-dependent metabolite trimethylamine-N-oxide, betaine and choline with non-alcoholic fatty liver disease in adults. Sci Rep. 2016;6:19076.

170. Leon-Mimila P, Villamil-Ramirez H, Li XS, et al. Trimethylamine N-oxide levels are associated with NASH in obese subjects with type 2 diabetes. Diabetes Metab. 2020.

171. Willeit P, Skroblin P, Kiechl S, Fernández-Hernando C, Mayr M. Liver microRNAs: potential mediators and biomarkers for metabolic and cardiovascular disease? Eur Heart J. 2016;37(43):3260-3266.

172. Virtue A, Johnson C, Lopez-Pastraña J, et al. MicroRNA-155 Deficiency Leads to Decreased Atherosclerosis, Increased White Adipose Tissue Obesity, and Nonalcoholic Fatty Liver Disease: A NOVEL MOUSE MODEL OF OBESITY PARADOX. J Biol Chem. 2017;292(4):1267-1287.

173. Ma Y, Ordovas JM. The integration of epigenetics and genetics in nutrition research for CVD risk factors. Proc Nutr Soc. 2017;76(3):333-346.

174. Zhang Z, Gao W, Long QQ, et al. Increased plasma levels of IncRNA H19 and LIPCAR are associated with increased risk of coronary artery disease in a Chinese population. Sci Rep. 2017;7(1):7491.

175. Kim A, Krishnan A, Hamilton JP, Woreta TA. The Impact of Dietary Patterns and Nutrition in Nonalcoholic Fatty Liver Disease. Gastroenterol Clin North Am. 2021;50(1):217-241.

176. Yaskolka Meir A, Rinott E, Tsaban G, et al. Effect of green-Mediterranean diet on intrahepatic fat: the DIRECT PLUS randomised controlled trial. Gut. 2021. 
177. Bian H, Hakkarainen A, Lundbom N, Yki-Jarvinen H. Effects of dietary interventions on liver volume in humans. Obesity (Silver Spring). 2014;22(4):989-995.

178. Colles SL, Dixon JB, Marks P, Strauss BJ, O'Brien PE. Preoperative weight loss with a very-low-energy diet: quantitation of changes in liver and abdominal fat by serial imaging. Am J Clin Nutr. 2006;84(2):304-311.

179. Hollingsworth KG, Abubacker MZ, Joubert I, Allison ME, Lomas DJ. Low-carbohydrate diet induced reduction of hepatic lipid content observed with a rapid non-invasive MRI technique. Br J Radiol. 2006;79(945):712-715.

180. Schutte S, Esser D, Hoevenaars FPM, et al. A 12-wk whole-grain wheat intervention protects against hepatic fat: the Graandioos study, a randomized trial in overweight subjects. Am J Clin Nutr. 2018;108(6):1264-1274.

181. Moore JB. Non-alcoholic fatty liver disease: the hepatic consequence of obesity and the metabolic syndrome. Proc Nutr Soc. 2010;69(2):211-220.

182. Oscarsson J, Onnerhag K, Riserus U, et al. Effects of free omega-3 carboxylic acids and fenofibrate on liver fat content in patients with hypertriglyceridemia and nonalcoholic fatty liver disease: A double-blind, randomized, placebo-controlled study. J Clin Lipidol. 2018;12(6):1390-1403 e1394.

183. Koliaki C, Szendroedi J, Kaul K, et al. Adaptation of hepatic mitochondrial function in humans with non-alcoholic fatty liver is lost in steatohepatitis. Cell Metab. 2015;21(5):739-746.

184. Van Name MA, Savoye M, Chick JM, et al. A Low omega-6 to omega-3 PUFA Ratio (n6:n-3 PUFA) Diet to Treat Fatty Liver Disease in Obese Youth. J Nutr. 2020;150(9):2314-2321.

185. Thoma C, Day CP, Trenell MI. Lifestyle interventions for the treatment of nonalcoholic fatty liver disease in adults: a systematic review. J Hepatol. 2012;56(1):255266.

186. Sevastianova K, Kotronen A, Gastaldelli A, et al. Genetic variation in PNPLA3 (adiponutrin) confers sensitivity to weight loss-induced decrease in liver fat in humans. Am J Clin Nutr. 2011;94(1):104-111. 
187. Luukkonen PK, Dufour S, Lyu K, et al. Effect of a ketogenic diet on hepatic steatosis and hepatic mitochondrial metabolism in nonalcoholic fatty liver disease. Proc Natl Acad Sci U S A. 2020;117(13):7347-7354.

188. Dongiovanni P, Valenti L. A Nutrigenomic Approach to Non-Alcoholic Fatty Liver Disease. Int J Mol Sci. 2017;18(7).

189. Meroni M, Longo M, Rustichelli A, Dongiovanni P. Nutrition and Genetics in NAFLD: The Perfect Binomium. Int J Mol Sci. 2020;21(8).

190. Scorletti E, West AL, Bhatia L, et al. Treating liver fat and serum triglyceride levels in NAFLD, effects of PNPLA3 and TM6SF2 genotypes: Results from the WELCOME trial. J Hepatol. 2015;63(6):1476-1483.

191. Nobili V, Bedogni G, Donati B, Alisi A, Valenti L. The I148M variant of PNPLA3 reduces the response to docosahexaenoic acid in children with non-alcoholic fatty liver disease. J Med Food. 2013;16(10):957-960.

192. Santoro N, Savoye M, Kim G, et al. Hepatic fat accumulation is modulated by the interaction between the rs738409 variant in the PNPLA3 gene and the dietary omega6/omega3 PUFA intake. PLoS One. 2012;7(5):e37827.

193. Cicero AFG, Colletti A, Bellentani S. Nutraceutical Approach to Non-Alcoholic Fatty Liver Disease (NAFLD): The Available Clinical Evidence. Nutrients. 2018;10(9).

194. Fogacci F, Banach M, Cicero AFG. Resveratrol effect on patients with non-alcoholic fatty liver disease: A matter of dose and treatment length. Diabetes Obes Metab. 2018;20(7):1798-1799.

195. Meroni M, Longo M, Dongiovanni P. The Role of Probiotics in Nonalcoholic Fatty Liver Disease: A New Insight into Therapeutic Strategies. Nutrients. 2019;11(11).

196. Aron-Wisnewsky J, Warmbrunn MV, Nieuwdorp M, Clement K. Nonalcoholic Fatty Liver Disease: Modulating Gut Microbiota to Improve Severity? Gastroenterology. 2020;158(7):1881-1898.

197. Parnell JA, Raman M, Rioux KP, Reimer RA. The potential role of prebiotic fibre for treatment and management of non-alcoholic fatty liver disease and associated obesity and insulin resistance. Liver Int. 2012;32(5):701-711.

198. Bakhshimoghaddam F, Shateri K, Sina M, Hashemian M, Alizadeh M. Daily Consumption of Synbiotic Yogurt Decreases Liver Steatosis in Patients with 
Nonalcoholic Fatty Liver Disease: A Randomized Controlled Clinical Trial. J Nutr. 2018;148(8):1276-1284.

199. Cai Y, Folkerts J, Folkerts G, Maurer M, Braber S. Microbiota-dependent and independent effects of dietary fibre on human health. $\mathrm{Br} J$ Pharmacol. 2020;177(6):1363-1381.

200. Da Silva HE, Teterina A, Comelli EM, et al. Nonalcoholic fatty liver disease is associated with dysbiosis independent of body mass index and insulin resistance. Sci Rep. 2018;8(1):1466.

201. Polyzos SA, Kang ES, Boutari C, Rhee EJ, Mantzoros CS. Current and emerging pharmacological options for the treatment of nonalcoholic steatohepatitis. Metabolism. 2020;111S:154203.

202. Zhou G, Myers R, Li Y, et al. Role of AMP-activated protein kinase in mechanism of metformin action. J Clin Invest. 2001;108(8):1167-1174.

203. Rena G, Hardie DG, Pearson ER. The mechanisms of action of metformin. Diabetologia. 2017;60(9):1577-1585.

204. Brandt A, Hernandez-Arriaga A, Kehm R, et al. Metformin attenuates the onset of nonalcoholic fatty liver disease and affects intestinal microbiota and barrier in small intestine. Sci Rep. 2019;9(1):6668.

205. Sun L, Xie C, Wang G, et al. Gut microbiota and intestinal FXR mediate the clinical benefits of metformin. Nat Med. 2018;24(12):1919-1929.

206. Delzenne NM, Cani PD, Everard A, Neyrinck AM, Bindels LB. Gut microorganisms as promising targets for the management of type 2 diabetes. Diabetologia. 2015;58(10):2206-2217.

207. de la Cuesta-Zuluaga J, Mueller NT, Corrales-Agudelo V, et al. Metformin Is Associated With Higher Relative Abundance of Mucin-Degrading Akkermansia muciniphila and Several Short-Chain Fatty Acid-Producing Microbiota in the Gut. Diabetes Care. 2017;40(1):54-62.

208. Ferri N, Corsini A, Sirtori C, Ruscica M. PPAR-alpha agonists are still on the rise: an update on clinical and experimental findings. Expert Opin Investig Drugs. 2017;26(5):593-602. 
209. Sasaki Y, Asahiyama M, Tanaka T, et al. Pemafibrate, a selective PPARalpha modulator, prevents non-alcoholic steatohepatitis development without reducing the hepatic triglyceride content. Sci Rep. 2020;10(1):7818.

210. Shinozaki S, Tahara T, Lefor AK, Ogura M. Pemafibrate decreases markers of hepatic inflammation in patients with non-alcoholic fatty liver disease. Clin Exp Hepatol. 2020;6(3):270-274.

211. Pradhan AD, Paynter NP, Everett BM, et al. Rationale and design of the Pemafibrate to Reduce Cardiovascular Outcomes by Reducing Triglycerides in Patients with Diabetes (PROMINENT) study. Am Heart J. 2018;206:80-93.

212. Bril F, Kalavalapalli S, Clark VC, et al. Response to Pioglitazone in Patients With Nonalcoholic Steatohepatitis With vs Without Type 2 Diabetes. Clin Gastroenterol Hepatol. 2018;16(4):558-566 e552.

213. Zhou Y, Huang Y, Ji X, Wang X, Shen L, Wang Y. Pioglitazone for the Primary and Secondary Prevention of Cardiovascular and Renal Outcomes in Patients with or at High Risk of Type 2 Diabetes Mellitus: A Meta-Analysis. J Clin Endocrinol Metab. 2020;105(5).

214. Ratziu V, Charlotte F, Bernhardt C, et al. Long-term efficacy of rosiglitazone in nonalcoholic steatohepatitis: results of the fatty liver improvement by rosiglitazone therapy (FLIRT 2) extension trial. Hepatology. 2010;51(2):445-453.

215. Belfort R, Harrison SA, Brown K, et al. A placebo-controlled trial of pioglitazone in subjects with nonalcoholic steatohepatitis. N Engl J Med. 2006;355(22):2297-2307.

216. Sanyal AJ, Chalasani N, Kowdley KV, et al. Pioglitazone, vitamin E, or placebo for nonalcoholic steatohepatitis. N Engl J Med. 2010;362(18):1675-1685.

217. Murphy CE, Rodgers PT. Effects of thiazolidinediones on bone loss and fracture. Ann Pharmacother. 2007;41(12):2014-2018.

218. Hattersley AT, Thorens B. Type 2 Diabetes, SGLT2 Inhibitors, and Glucose Secretion. N Engl J Med. 2015;373(10):974-976.

219. Dokmak A, Almeqdadi M, Trivedi H, Krishnan S. Rise of sodium-glucose cotransporter 2 inhibitors in the management of nonalcoholic fatty liver disease. World J Hepatol. 2019;11(7):562-573. 
220. Osataphan S, Macchi C, Singhal G, et al. SGLT2 inhibition reprograms systemic metabolism via FGF21-dependent and -independent mechanisms. JCI Insight. $2019 ; 4(5)$.

221. Morax S, Herdan ML. [Palpebral wounds and lacerations]. Rev Prat. 1987;37(37):2191-2198.

222. Zhang $\mathrm{XL}$, Zhu QQ, Chen $\mathrm{YH}$, et al. Cardiovascular Safety, Long-Term Noncardiovascular Safety, and Efficacy of Sodium-Glucose Cotransporter 2 Inhibitors in Patients With Type 2 Diabetes Mellitus: A Systemic Review and Meta-Analysis With Trial Sequential Analysis. J Am Heart Assoc. 2018;7(2).

223. Drucker DJ. Mechanisms of Action and Therapeutic Application of Glucagon-like Peptide-1. Cell Metab. 2018;27(4):740-756.

224. Newsome PN, Buchholtz K, Cusi K, et al. A Placebo-Controlled Trial of Subcutaneous Semaglutide in Nonalcoholic Steatohepatitis. N Engl J Med. 2020.

225. Vella A. Mechanism of action of DPP-4 inhibitors--new insights. J Clin Endocrinol Metab. 2012;97(8):2626-2628.

226. Kawakubo M, Tanaka M, Ochi K, et al. Dipeptidyl peptidase-4 inhibition prevents nonalcoholic steatohepatitis-associated liver fibrosis and tumor development in mice independently of its anti-diabetic effects. Sci Rep. 2020;10(1):983.

227. Fukuhara T, Hyogo H, Ochi $\mathrm{H}$, et al. Efficacy and safety of sitagliptin for the treatment of nonalcoholic fatty liver disease with type 2 diabetes mellitus. Hepatogastroenterology. 2014;61(130):323-328.

228. Cui J, Philo L, Nguyen P, et al. Sitagliptin vs. placebo for non-alcoholic fatty liver disease: A randomized controlled trial. J Hepatol. 2016;65(2):369-376.

229. Wang YD, Chen WD, Wang M, Yu D, Forman BM, Huang W. Farnesoid X receptor antagonizes nuclear factor kappaB in hepatic inflammatory response. Hepatology. 2008;48(5):1632-1643.

230. Kong B, Luyendyk JP, Tawfik O, Guo GL. Farnesoid X receptor deficiency induces nonalcoholic steatohepatitis in low-density lipoprotein receptor-knockout mice fed a high-fat diet. J Pharmacol Exp Ther. 2009;328(1):116-122.

231. Han CY. Update on FXR Biology: Promising Therapeutic Target? Int J Mol Sci. 2018;19(7). 
232. Mudaliar S, Henry RR, Sanyal AJ, et al. Efficacy and safety of the farnesoid X receptor agonist obeticholic acid in patients with type 2 diabetes and nonalcoholic fatty liver disease. Gastroenterology. 2013;145(3):574-582 e571.

233. Nevens F, Andreone P, Mazzella G, et al. A Placebo-Controlled Trial of Obeticholic Acid in Primary Biliary Cholangitis. N Engl J Med. 2016;375(7):631-643.

234. Neuschwander-Tetri BA, Loomba R, Sanyal AJ, et al. Farnesoid X nuclear receptor ligand obeticholic acid for non-cirrhotic, non-alcoholic steatohepatitis (FLINT): a multicentre, randomised, placebo-controlled trial. Lancet. 2015;385(9972):956-965.

235. Younossi ZM, Ratziu V, Loomba R, et al. Obeticholic acid for the treatment of nonalcoholic steatohepatitis: interim analysis from a multicentre, randomised, placebocontrolled phase 3 trial. Lancet. 2019;394(10215):2184-2196.

236. Carino A, Biagioli M, Marchiano S, et al. Opposite effects of the FXR agonist obeticholic acid on Mafg and Nrf2 mediate the development of acute liver injury in rodent models of cholestasis. Biochim Biophys Acta Mol Cell Biol Lipids. 2020;1865(9):158733.

237. Cannon CP, Blazing MA, Giugliano RP, et al. Ezetimibe Added to Statin Therapy after Acute Coronary Syndromes. N Engl J Med. 2015;372(25):2387-2397.

238. Tanaka Y, Ikeda T, Ogawa H, Kamisako T. Ezetimibe Markedly Reduces Hepatic Triglycerides and Cholesterol in Rats Fed on Fish Oil by Increasing the Expression of Cholesterol Efflux Transporters. J Pharmacol Exp Ther. 2020;374(1):175-183.

239. Simon TG, Corey KE, Chung RT, Giugliano R. Cardiovascular Risk Reduction in Patients with Nonalcoholic Fatty Liver Disease: The Potential Role of Ezetimibe. Dig Dis Sci. 2016;61(12):3425-3435.

240. Altmann SW, Davis HR, Jr., Zhu LJ, et al. Niemann-Pick C1 Like 1 protein is critical for intestinal cholesterol absorption. Science. 2004;303(5661):1201-1204.

241. Xavier BM, Zein AA, Venes A, Wang J, Lee JY. Transmembrane Polar Relay Drives the Allosteric Regulation for ABCG5/G8 Sterol Transporter. Int J Mol Sci. 2020;21(22).

242. Toyoda Y, Takada T, Umezawa M, et al. Identification of hepatic NPC1L1 as an NAFLD risk factor evidenced by ezetimibe-mediated steatosis prevention and recovery. FASEB Bioadv. 2019;1(5):283-295.

243. Ruscica M, Corsini A, Ferri N, Banach M, Sirtori CR. Clinical approach to the inflammatory etiology of cardiovascular diseases. Pharmacol Res. 2020;159:104916. 
244. Jonas W, Schurmann A. Genetic and epigenetic factors determining NAFLD risk. Mol Metab. 2020:101111.

This article is protected by copyright. All rights reserved 
Table 1. Dominant genetic modifiers known to shape both NAFLD susceptibility and progression.

\begin{tabular}{|c|c|c|c|}
\hline Gene & Liver cell type & Function & CAD risk \\
\hline & - Hepatocytes & - Lipid droplet remodeling & - reduced \\
\hline PNPLA3 & - Stellate cells & $\begin{array}{l}\text { - Modulation of retinol production and } \\
\text { release }\end{array}$ & OR: $0.92(95 \% \mathrm{Cl} 0.87-0.97)$ \\
\hline TM6SF2 & Hepatocytes & $\begin{array}{l}\text { - VLDL }{ }_{1} \text {-TG production was } 35 \% \text { lower in } \\
\text { homozygous TM6SF2 E167K carriers }\end{array}$ & $\begin{array}{l}\text { - reduced } \\
\text { OR: } 0.78(95 \% \mathrm{Cl} 0.65-0.93)\end{array}$ \\
\hline MBOAT7 & $\begin{array}{l}\text { - Hepatocytes } \\
\text { - Hepatic sinusoidal cells } \\
\text { - Stellate cells }\end{array}$ & - Remodeling of phosphatidylinositol & $\begin{array}{l}\text { - neutral effect } \\
\text { (OR: } 1.01 \text { (0.37-1.05) } \\
\text { - raised risk of venous } \\
\text { thromboembolism }\end{array}$ \\
\hline HSD17B13 & - Hepatocytes & $\begin{array}{l}\text { - Lipid droplet remodeling } \\
\text { - Retinol metabolism }\end{array}$ & n.a. \\
\hline GCKR & - Hepatocytes & $\begin{array}{l}\text { - Raised glycolytic flux } \\
\text { - Regulation of de novo lipogenesis }\end{array}$ & n.a. \\
\hline
\end{tabular}


HSD17B13, Hydroxysteroid 17-Beta Dehydrogenase 13; MBOAT7, Membrane Bound O-acyltransferase Domain-containing 7; NAFLD, nonalcoholic fatty liver disease; PNPLA3, Patatin-like Phospholipase Domain-Containing 3; TM6SF2, Transmembrane 6 Superfamily Member 2 gene. Adapted with permission of Elsevier ${ }^{244}$. 


\section{Figure legend}

Figure 1. Proposed criteria for the clinical diagnosis of MAFLD (with permission of Elsevier ${ }^{14}$ ).

Figure 2. Contribution of NAFLD to the develpoment of cardiovascular disease. The mechanisms underlying NAFLD pathogenesis are multifactorial. Parallel hits as environment, gut-microbiota, genetics and epigenetics participate to the disease onset and progression. The first step in NAFLD development is hepatic fat infiltration, mainly due to insulin resistance, which leads to adipose tissue lipolysis with the consequent efflux of free fatty acids to the liver. In addition, the compensatory hyperinsulinemia activates the de novo lipogenesis, trough SREBP1c, and exacerbates fat accumulation. The adipose tissue releases adipokines, such as leptin, adiponectin, TNF- $\alpha$ and IL-6. Excess fat is cleared from the liver by an enhanced lipoprotein secretion and mitochondrial $\beta$-oxidation. The latter results in increased reactive-oxigen species production leading to the activation of inflammatory pathways. The endothelial dysfunction and lipid accumulation in arteries is linked to inflammation, which results in lipid accumulation in macrophages that are called "foam cells". This leads to alteration of intima-media thickness and plaques formation. IL, interleukin; Hydroxysteroid 17-Beta Dehydrogenase 13 (HSD17B13); Membrane Bound O-acyltransferase Domain-containing 7 (MBOAT7); NAFLD, nonalcoholic fatty liver disease; NASH, Nonalcoholic steatohepatitis; TNF, tumor necrosis factor; Patatin-like Phospholipase Domain-Containing 3 (PNPLA3), SREBP1c, sterol regulatory element-binding protein; Transmembrane 6 Superfamily Member 2 gene (TM6SF2).

Figure 3. Impact of genetic risk variants on liver damage. Comparison of the impact of risk variants PNPLA3 I148M (rs738409), TM6SF2 E167K (rs58542926), GCKR P446L (rs1260326) and MBOAT7 rs641738 on hepatic fat vs. liver damage. Panel a) histological steatosis vs. ballooning; panel b) histological steatosis vs. necroinflammation; panel c) histological steatosis vs. fibrosis; panel d) hepatic fat content vs. serum ALT levels. Reproduced with permission of Wiley ${ }^{23}$.

Figure 4. Pharmalological approaches which reduce CV risk and may potentially be used in NAFLD patients. Metformin inhibits AMPK pathway; Pemafibrate activates PPAR $\alpha$ and Pioglitazone 
activates PPAR $\gamma$; Obeticholic acid inhibites FXR; Liraglutide, exenatide, dulaglutide and senaglutide are GLP-1R agonist; Sitagliptin, saxagliptin, linagliptin, alogliptin, and vidagliptin are DPP-4 inhibitors; Glifozines are SGLT-2 inhibitors. CV, cardiovascular; GLP-1R, glucagon-like peptide 1; DDP-4, dideptidylpeptidase-4. NAFLD, nonalcoholic fatty liver disease; CVD, cardiovascular disease. 


\section{Figure 2}

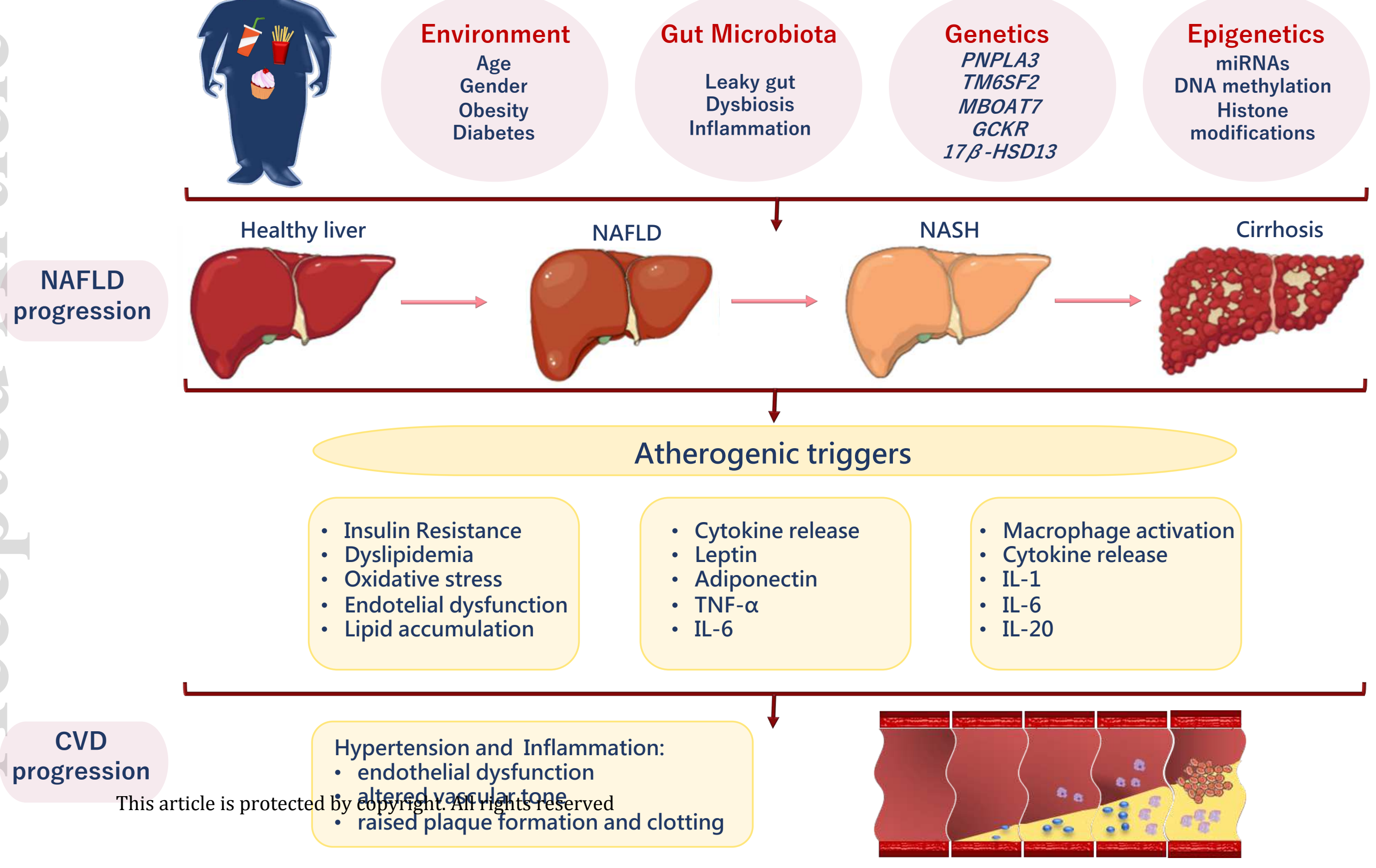




\section{Figure 3}
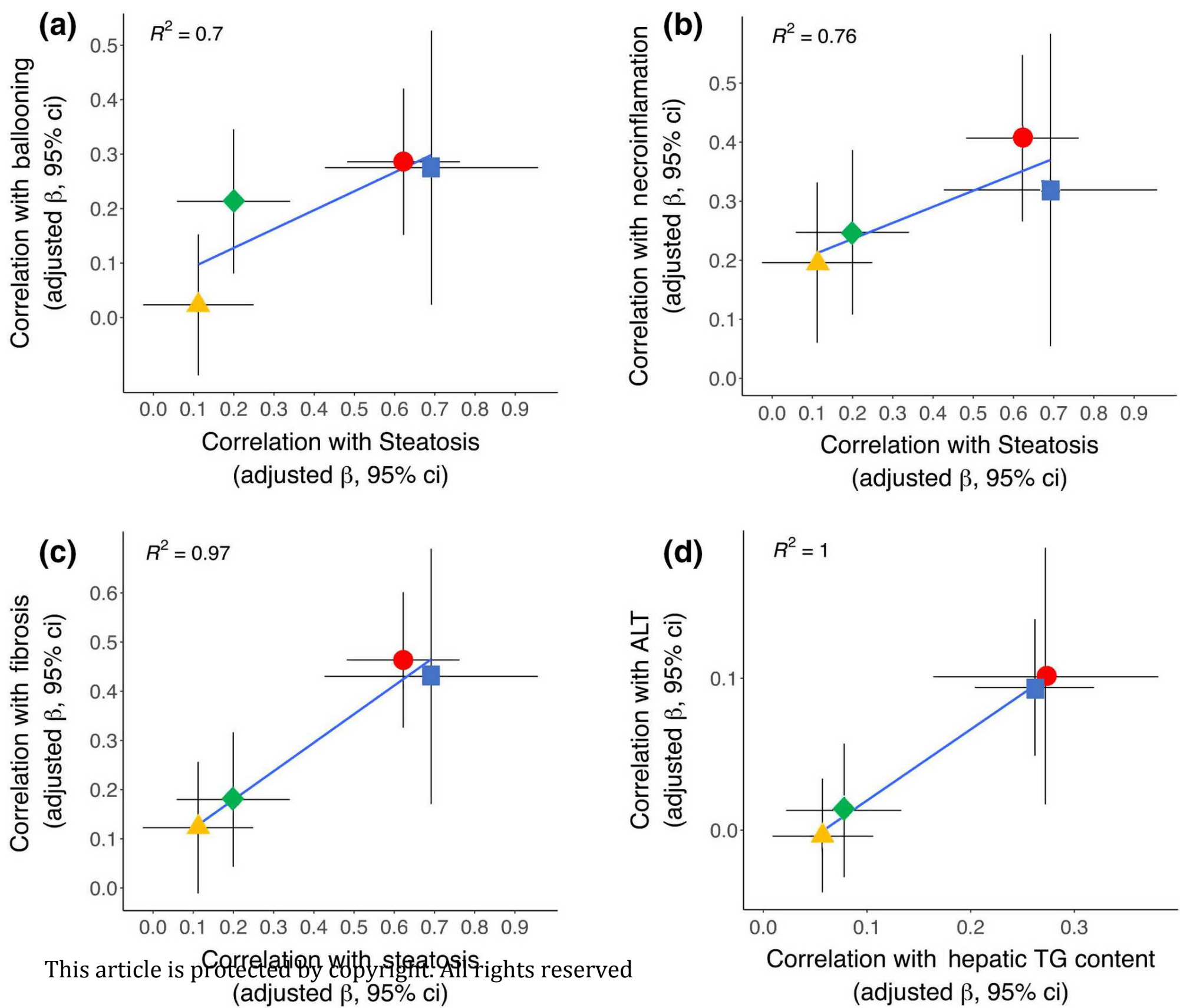

(adjusted $\beta, 95 \%$ ci) 


\section{Figure 4}

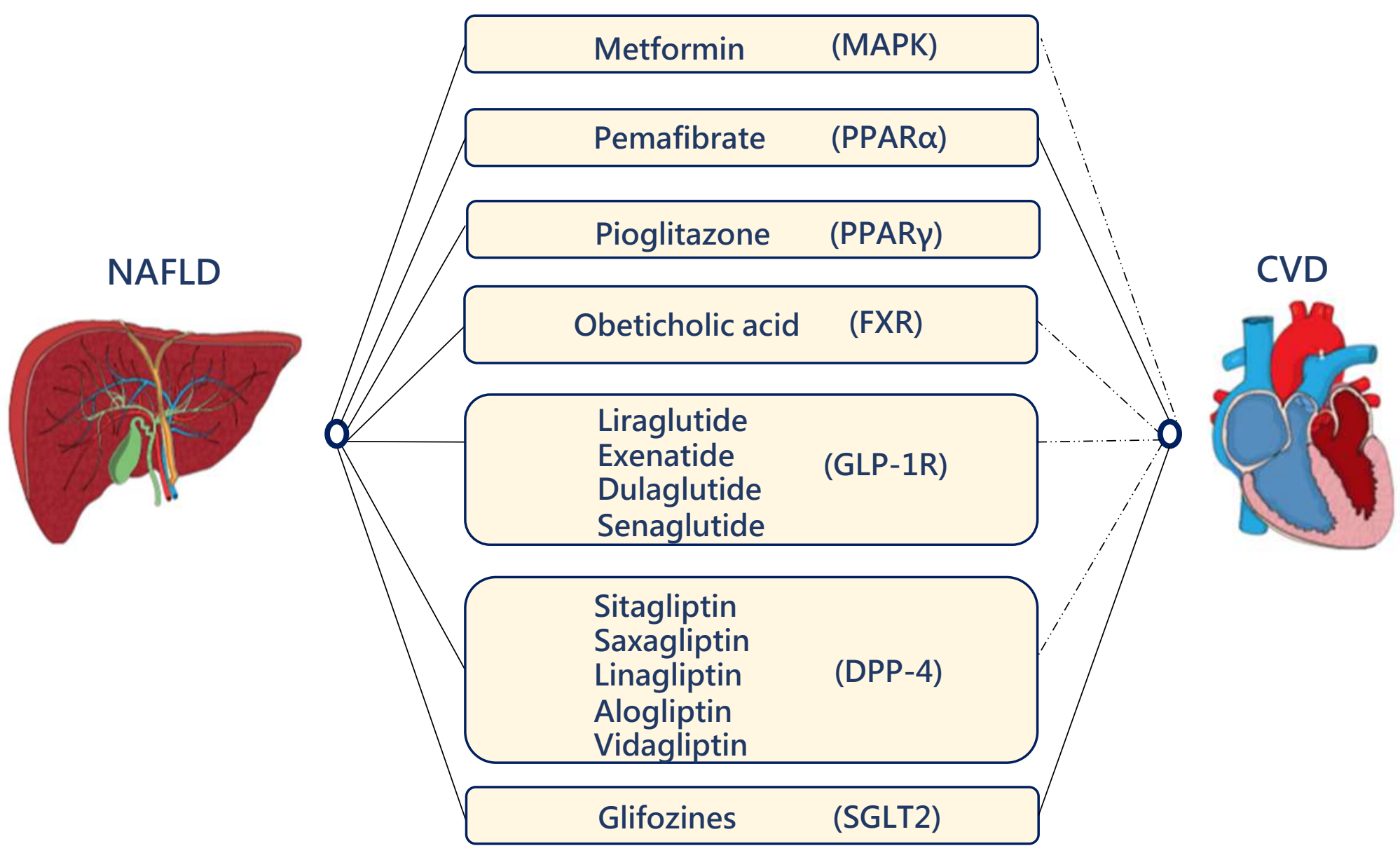

This article is protected by copyright. All rights reserved 